\title{
16. PETROLOGY AND GEOCHEMISTRY OF VOLCANIC ROCKS FROM THE NEW HEBRIDES FOREARC REGION, SITES 827, 829, AND 830 ${ }^{1}$
}

\author{
M. Coltorti, ${ }^{2}$ P.E. Baker, ${ }^{3}$ L. Briqueu, ${ }^{4}$ T. Hasenaka,${ }^{5}$ and B. Galassi ${ }^{2}$
}

\begin{abstract}
Igneous rocks recovered from Ocean Drilling Program (ODP) Leg 134 Sites 827, 829, and 830 at the toe of the forearc slope of New Hebrides Island Arc were investigated, using petrography, mineral chemistry, major and trace element, and $\mathrm{Sr}$, Nd, and $\mathrm{Pb}$ isotopic analyses.

Basaltic and andesitic clasts, together with detrital crystals of plagioclase, pyroxenes, and amphiboles embedded in sed-lithic conglomerate or volcanic siltstone and sandstone of Pleistocene age, were recovered from Sites 827 and 830 . Petrological features of these lava clasts suggest a provenance from the Western Belt of New Hebrides Island Arc; igneous constituents were incorporated into breccias and sandstones, which were in turn reworked into a second generation breccia.

Drilling at Site 829 recovered a variety of igneous rocks including basalts and probably comagmatic dolerites and gabbros, plus rare ultramafic rocks. Geochemical features, including $\mathrm{Pb}$ isotopic ratios, of the mafic rocks are intermediate between midocean ridge basalts and island arc tholeites, and these rocks are interpreted to be backarc basin basalts. No correlates of these mafic rocks are known from Espiritu Santo and Malakula islands, nor do they occur in the Pleistocene volcanic breccias at Sites 827 and 830 . However, basalts with very similar trace element and isotopic compositions have been recovered from the northern flank of North d'Entrecasteaux Ridge at Site 828. It is proposed that igneous rocks drilled at Site 829 represent material from the North d'Entrecasteaux Ridge accreted onto the over-riding Pacific Plate during collision.

An original depleted mantle harzburgitic composition is inferred for a serpentinite clast recovered at 407 meters below seafloor (mbsf) in Hole 829A. Its provenance is a matter of speculation. It could have been brought up along a deep thrust fault affecting the Pacific Plate at the colliding margin, or analogous to the Site 829 basaltic lavas, it may represent material accreted from the North d'Entrecasteaux Ridge.
\end{abstract}

\section{INTRODUCTION}

One of the most controversial aspects of plate tectonics is the mode of interaction between plates at convergent margins. Definition of the mass balance between material added to the over-riding plate and that carried down into the mantle by subduction has attracted the interest of many Earth scientists.

Where convergent compressional tectonics operate, accretion is active and formation of an accretionary wedge occurs with consequent seaward migration of the trench (Karig and Sharman, 1975). In contrast, arc erosion and sediment subduction, with consequent arcward migration of the trench occur at extensional convergent plate boundary settings (Scholl et al., 1980; Aubouin, 1989). Interaction between the converging plates is more complicated where a structural high, such as a continuous ridge (Kyushu-Palau Ridge in the Nankai Trough, Yamazaki and Okamura, 1989; Lousville Ridge in the Tonga Trench, Ballance et al., 1989) or a seamount (Daiichi Kashima seamount in the Japan Trench, Lallemand et al., 1989; Kobayashi et al., 1987; Yamazaki and Okamura, 1989), collides with an arc and begins to be subducted. In this case, a major question concerns whether the topographic high will be accreted or subducted.

The collision and subduction of the d'Entrecasteaux Zone (DEZ) with the Western Belt islands (Espiritu Santo and Malakula) of the New Hebrides Island Arc (Fig. 1) provides an excellent opportunity to study a ridge/seamount collision with an arc (Collot and Fisher,

\footnotetext{
${ }^{1}$ Greene, H.G., Collot, J.-Y., Stokking, L.B., et al., 1994. Proc. ODP, Sci. Results, 134: College Station, TX (Ocean Drilling Program).

${ }^{2}$ Institute of Mineralogy, University of Ferrara, C.so E. $1^{\circ}$ d'Este, 32, 44100 Ferrara, Italy.

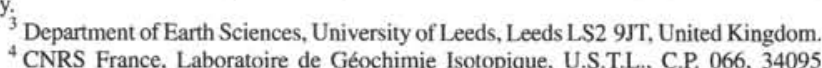
Montpellier Cedex 2, France.

5 Institute of Mineralogy, Petrology and Economic Geology, Faculty of Science, Tohoku University, Aoba, Sendai, Miyagi 980, Japan.
}

1988; Fisher et al., 1991). The northern continuous ridge (North d'Entrecasteaux Ridge [NDR]) and a parallel southern chain of seamounts (Southern d'Entrecasteaux Chain [SDC]), which constitute the two parts of the DEZ, caused different styles of collisional deformation of the forearc slope. The collision of the SDC resulted in large anticlines, faults, and thrust faults, dipping gently eastward, together with an arc-slope indentation of about $10 \mathrm{~km}$. In contrast, the collision of the NDR with the western slope of Espiritu Santo Island created a broad shallow protrusion, Wousi Bank, on the forearc region, almost reaching sea level (Collot et al., 1992, this volume; Greene et al., this volume).

Reconstruction of the stratigraphic sequences in the drilled sites on the arc slope, and comparison with rocks drilled on the NDR (Site 828; Coltorti et al., this volume) and Bougainville Guyot (Site 831; Baker et al., this volume) allow definition of the deformation style of these two regions and the accretionary processes transferring material from the Australia-India Plate to the Pacific Plate.

Petrography, mineral chemistry, major and trace element analyses, and $\mathrm{Sr}, \mathrm{Nd}$, and $\mathrm{Pb}$ isotopic determinations on igneous clasts recovered from Sites 827,829 , and 830 are used to distinguish between the material derived from the arc and that scraped off the DEZ by ridge/ seamount collision and subduction. This information will contribute to a better understanding of the structural evolution of this forearc region.

\section{SITE LOCATION AND STRATIGRAPHY}

Three sites (Sites 827,829 , and 830 ) on the forearc slope were chosen to investigate the geological and tectonic effects of ridge/ seamount collision with the New Hebrides Island Arc (Fig. 1).

Site $827\left(15^{\circ} 17.75^{\prime} \mathrm{S}, 166^{\circ} 21.11^{\prime} \mathrm{E}\right)$ is located on a flat terrace-like feature along the northern flank of Wousi Bank at a depth of 2803.4 mbsl; it is just in front of the NDR, $4 \mathrm{~km}$ east of the trace of the trench, and about $35 \mathrm{~km}$ west of the western shore of Espiritu Santo Island. Holes $827 \mathrm{~A}$ and $827 \mathrm{~B}$ were drilled at this site, coring 110.6 and $400.4 \mathrm{~m}$, respectively. Four lithostratigraphic units were recognized. 


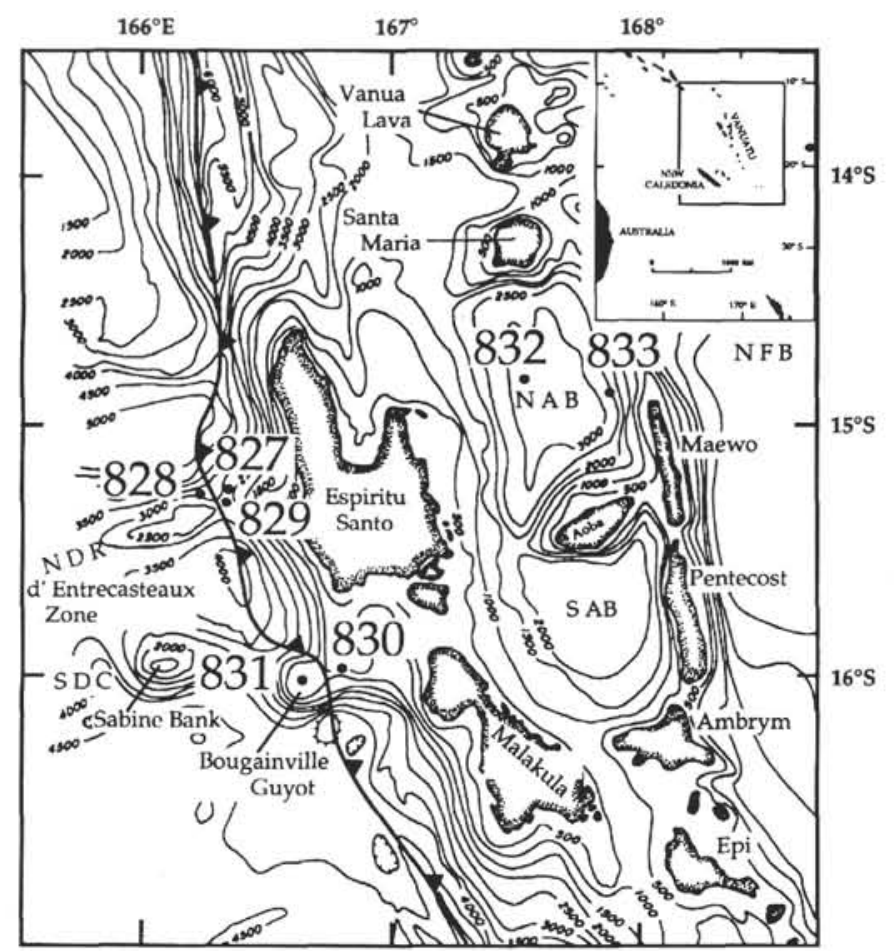

Figure 1. Location of Leg 134 sites. NDR = North d'Entrecasteaux Ridge; SDC $=$ South d'Entrecasteaux Chain; $\mathrm{NAB}=$ North Aoba Basin; $\mathrm{SAB}=$ South $\mathrm{Aoba}$ Basin; NFB = North Fiji Basin. Bold line with teeth indicates plate boundary; teeth are on upper plate. Bathymetry in meters.

Units I and II are a sequence of upper Pliocene to Pleistocene volcanic silt and siltstone with variable clay and sand components. Unit III contains upper Pliocene to middle Pleistocene highly bioturbated calcareous volcanic siltstone with intervals of sed-lithic conglomerate. Lithostratigraphic Unit IV (252.6-400.4 mbsf) is little understood because of poor recovery, and its age is unknown because samples were barren of fossils. This unit mainly consists of sed-lithic conglomerate, very well-lithified volcanic siltstone, and sandstone containing mainly igneous rock fragments and crystals. Clasts, ranging in size from pebbles to the maximum diameter that can fit in the core barrel, are very angular to rounded and poorly sorted. Igneous fragments are mainly represented by volcanic breccia, andesites, and less commonly, dacites; detrital crystals include plagioclases, pyroxenes, and amphiboles.

Site $829\left(15^{\circ} 18.97^{\prime} \mathrm{S}, 166^{\circ} 20.70^{\prime} \mathrm{E}\right)$ is located within the collision zone of DEZ along the forearc slope of the New Hebrides Island Arc, where the NDR impinges upon the arc slope, about $3 \mathrm{~km}$ south of Site 827. Holes $829 \mathrm{~A}, \mathrm{~B}$, and $\mathrm{C}$ were drilled at this site; $590 \mathrm{~m}$ were cored in the first hole, whereas the other two recovered undisturbed sediments from the first $100 \mathrm{~m}$ of the sequence. The lithostratigraphy of Site 829 is complicated by frequent repetitions of the sequence caused by thrust-faults. 21 lithostratigraphic units were distinguished and divided into four composite units. A large variety of effusive, intrusive, and subvolcanic igneous rocks was found. These rocks mainly occur as clasts in volcanic breccia or sed-lithic breccia, and poor recovery often made it difficult to determine whether gabbros were derived from a homogeneous body or from a volcanic breccia. Igneous rocks were encountered at two levels in the stratigraphic sequence. The first, overlying middle Oligocene calcareous chalk, is found between 397.7 and 416.6 mbsf (Cores 134-829A-43R and -44R, Unit VII) for a total recovery of $7 \mathrm{~m}$. The second, underlying Pliocene-Pleistocene sandy volcanic siltstone, is between $513.5 \mathrm{mbsf}$ and the bottom of the hole (581.2 mbsf, Unit XVI), with a recovery of about $14 \mathrm{~m}$ (for a complete stratigraphic report, see Meschede and Pelletier, this volume, and Reid et al., this volume). K/Ar age determinations on Samples 134-829A-
59R-1, 81-96 cm, and $-61 \mathrm{R}-1,36-38 \mathrm{~cm}$, give ages of $9.8 \pm 3 \mathrm{Ma}$ and $26.7 \pm 2 \mathrm{Ma}$, respectively (Rex, this volume).

Site $830\left(15^{\circ} 57.00^{\prime} \mathrm{S}, 166^{\circ} 46.79^{\prime} \mathrm{E}\right)$ is located on the forearc slope in the collision zone between Bougainville Guyot and the Central New Hebrides Island Arc, about $6.5 \mathrm{~km}$ east of the plate boundary and about $30 \mathrm{~km}$ south of the southern coast of Espiritu Santo Island. Holes $830 \mathrm{~A}, \mathrm{~B}$, and $\mathrm{C}$ were drilled at this site for a total penetration depth of $350.6 \mathrm{~m}$ and $122.05 \mathrm{~m}$ of recovery. Two major lithostratigraphic units were described. Unit I consists of Pleistocene volcanic silt and siltstone with variable amounts of sand and clay and lies unconformably upon lithostratigraphic Unit II, which is a sequence of altered, very coarse volcaniclastic sandstone, partially lithified and poorly sorted, with a matrix of sandy silt. Clasts and isolated pebbles of volcanic breccias and lavas were encountered only in Unit II. They were found at various levels: from 174.9 to $184.6 \mathrm{mbsf}$ (Core 134830B-14R) and from 252.4 to 262.2 mbsf (Core 134-830B-22R) in Hole $830 \mathrm{~B}$, and from 235.0 to $263.7 \mathrm{mbsf}$ (Cores $134-830 \mathrm{C}-1 \mathrm{R},-2 \mathrm{R}$, and $-3 R$ ) in Hole $830 \mathrm{C}$. All samples from lithostratigraphic Unit II were unfossiliferous.

\section{ANALYTICAL METHODS}

$\mathrm{X}$-ray fluorescence (XRF) major and trace element analyses (excluding rare earth elements [REE]) were conducted aboard the JOIDES Resolution, at the Institute of Mineralogy, Ferrara University (Italy), and at the Institute of Mineralogy, Petrology and Economic Geology, Tohoku University (Japan).

Analyses aboard ship were performed on glass discs for major elements and on powder pellets for trace elements, using a Compton scattering technique for matrix absorbing corrections (Reynolds, 1967). Major element data are considered accurate between $1 \%$ and $5 \%$, whereas accuracy for trace elements varies from $2 \%$ to $10 \%$, except for $\mathrm{Ba}$ and $\mathrm{Ce}$, which exceed $10 \%$ (see "Explanatory Notes," Collot, Greene, Stokking, et al., 1992).

Analyses from Ferrara University were performed on powder pellets using a wavelength-dispersive automated Philips PW 1400 spectrometer. Major elements were determined by a full matrix correction procedure (Franzini et al., 1975), whereas for trace elements, experimentally determined correction coefficients were used (Leoni and Saitta, 1976). Accuracy and precision for major elements are estimated better than $3 \%$ for $\mathrm{Si}, \mathrm{Ti}, \mathrm{Fe}, \mathrm{Ca}$, and $\mathrm{K}$ and $7 \%$ for $\mathrm{Mg}, \mathrm{Na}$, $\mathrm{Al}, \mathrm{Mn}$, and $\mathrm{P}$; for trace elements (above $10 \mathrm{ppm}$ ) they are better than $7 \%$ for $\mathrm{Rb}, \mathrm{Nb}, \mathrm{Y}, \mathrm{Sr}, \mathrm{V}$, and $15 \%$ for $\mathrm{Zr}, \mathrm{La}, \mathrm{Ce}, \mathrm{Ba}, \mathrm{Ni}, \mathrm{Co}$, and $\mathrm{Cr}$. Analyses of reference standards AGV 1 and BR are reported in Table 1 for comparison. Loss on ignition (LOI) was determined by a gravimetric method.

REE and Y were determined at the Centre de Recherches Pétrographiques et Géochimiques, Nancy (France), by inductively coupled plasma (ICP) emission spectrometry with an accuracy of $15 \%$ for $\mathrm{Yb}$ and $\mathrm{Lu}$ and better than $8 \%$ for the other REE (see analyses of reference standards from Roelandts and Michel, 1986). Analytical methods for analyses performed at Tohoku University (including REE) are described in Hasenaka et al. (this volume). In order to facilitate comparison between analyses from different laboratories, $\mathrm{Fe}_{2} \mathrm{O}_{3}$ was calculated as $0.15 \mathrm{FeO}$, and analyses were recalculated to $100 \%$ on an anhydrous basis. Analyses carried out in the different laboratories display reasonable agreement. Some discrepancies can be observed in the $\mathrm{SiO}_{2}$ and $\mathrm{CaO}$ contents and are probably related to variable degrees of alteration, even in samples very close together in the core (most of them as clasts). Trace elements, particularly those considered unaffected by secondary remobilization, show good agreement, except Y by ICP, which is systematically lower (about $15 \%$ ) than that by XRF. Isotopic analyses were carried out at the Laboratoire de Géochimique Isotopique, Université de Montpellier, after leaching using $2 \mathrm{~N} \mathrm{HF}+0.5 \mathrm{~N} \mathrm{HBr}$ mixture and cold $2.5 \mathrm{~N} \mathrm{HCl}$ (see the analytical methods section in Briqueu et al., this volume). Minerals were analyzed at the University of Leeds (UK) using a CAMECA SX-50 electron-probe microanalyzer fitted 
with three wavelength-dispersive spectrometers and a LINK 10/55S energy-dispersive system, at an accelerating voltage of $15 \mathrm{kV}$, and specimen current of $15 \mathrm{nA}$. Natural silicates and oxides standards were used, and the raw data were corrected using CAMECA proprietary software. For more details see the analytical methods section in Baker et al. (this volume).

\section{PETROGRAPHY}

\section{Site 827}

The majority of clasts encountered in lithostratigraphic Unit IV in Hole $827 \mathrm{~B}$ are lithified volcanic breccias or coarse sandstones containing a preponderance of igneous rock fragments and crystals. Clasts of andesite are set in a very fine-grained clay-chlorite matrix that possibly represents devitrified glass. The matrix also includes some calcite and sporadic foraminifers. The coarser constituents in the matrix include discrete subhedral crystals of plagioclase, clinopyroxene, amphiboles, and opaques that are similar in type and proportion to the phases in the rock fragments. Andesitic clasts are highly plagioclase-phyric with occasional clinopyroxenes and opaques in a fine-grained groundmass made up of plagioclase in association with secondary minerals including chlorite, carbonate, and clay. One clast has a slightly more evolved composition and is referred to as dacitic breccia. Alkali feldspar is more abundant than in the andesitic breccia and a small proportion of quartz (around $1 \%$ ) occurs in the groundmass.

\section{Site 829}

Clinopyroxene + plagioclase-phyric basalts, pyroxenites, and serpentinites were found in Unit VII between 397.7 and 416.6 mbsf (Cores 134-829A-43R and -44R), and plagioclase-phyric basalts, dolerites, microgabbros, and olivine gabbros were encountered in Unit XVI, between 513.5 and 581.2 mbsf (bottom of Hole 829A). Alteration is moderate, and veins and fractures, as well as vesicles, are relatively scarce. The secondary mineral assemblage is typical of low temperature submarine alteration. Chlorite and actinolite in basalts may be attributed to greenschist facies metamorphism, whereas the occurrence of green hornblende in gabbros probably reflects subsolidus deuteric alteration.

\section{Sparsely Clinopyroxene + Plagioclase-phyric Basalts}

These rocks are hypocrystalline, fine-grained, and vary from aphyric to sparsely phyric, containing microphenocrysts of subhedral, weakly zoned clinopyroxene $(0.1-0.5 \mathrm{~mm})$ and lath-shaped plagioclase $(0.2-0.6 \mathrm{~mm})$; a few olivine phenocrysts were found in only one instance (Sample 134-829A-43R-2, 145-147 cm). Groundmass is dominated by plagioclase microlites with intergranular clinopyroxene, opaques, and variable amounts of glass (intersertal to hyalopilitic textures). Elongate, low-crystallinity patches are constituted by brown, altered, devitrified glass. In this lithotype, vesicles are abundant (20-30 vol\%), irregularly distributed and infilled with green- to brown-colored, zonally arranged, mixed-layer clay minerals. Where vesicles are more concentrated, devitrified glass, sometimes with a well-developed spherulitic texture, dominates the groundmass.

\section{Moderately Plagioclase-phyric Basalts}

These are non-vesicular, hypocrystalline, and fine-grained rocks with scattered lath-shaped or tabular, weakly zoned, plagioclase microphenocrysts $(0.20-0.60 \mathrm{~mm})$. Unidentified mafic phases, totally replaced by chlorite and reddish mixed-layer clay minerals, sporadically occur in the phenocryst assemblage. The groundmass is hyalopilitic with skeletal $\mathrm{H}$-shaped plagioclase, rare acicular clinopyroxenes, and small oxide grains set in an altered, completely devitrified glassy mesostasis. Very thin $(0.03-0.06 \mathrm{~mm})$ veins are filled with oxides or zeolites. Rare, secondary actinolitic amphibole also occurs.

\section{Dolerites and Microgabbros}

Dolerites are medium-grained $(0.5-1 \mathrm{~mm})$ and comprised of plagioclase laths $(55 \%)$ surrounded by subequant clinopyroxene crystals $(40 \%)$ in a subophitic texture, which becomes locally ophitic when plagioclase crystals are embedded in large clinopyroxenes. Plagioclase is generally radially arranged and displays strong normal zoning, whereas clinopyroxene is colorless and nearly unzoned. Iron oxides (up to $5 \%$ ) are also present. Alteration products are chlorite and clay minerals. Green hornblende often rims clinopyroxene.

Microgabbros show an ophitic texture with modal proportions similar to dolerites, the main difference being the increase in grain size (1-3 mm).

\section{Gabbros}

These rocks are holocrystalline, medium- to coarse-grained (around $5 \mathrm{~mm}$ ), with subophitic to ophitic texture. Textural relationships suggest that the order of crystallization is olivine, plagioclase, clinopyroxene, and finally magnetite. The idiomorphic bladed crystals of plagioclase are fresh and strongly zoned. Olivine occurs as markedly zoned subhedral, partially altered crystals; when joined by plagioclase, the two phases show cotectic intergrowth. Clinopyroxene, which is sometimes intergrown with Ti-magnetite, occurs as small grains among plagioclase or as large, poikilitic, strongly-zoned crystals. They are often mantled by green hornblende, a feature which is rather common in gabbros dredged from oceanic settings (Prichard and Cann, 1982). Alteration products are iddingsite, and locally, calcite after olivine, and chlorite after clinopyroxene. Chlorite, calcite, clay minerals, and iron hydroxides fill rare cavities and veins. The presence of tiny flakes of biotite in a few samples is noted. This mineral is very unusual in ocean floor rocks and may indicate a relatively high $\mathrm{K}$ content compared to normal mid-ocean ridge basalts (Prichard and Cann, 1982; Spadea et al., 1991).

\section{Ultramafic Rocks}

The pyroxenite (Sample 134-829A-43R-1, 133-135 cm) has an allotriomorphic-granular texture and is composed of clinopyroxene $(80 \%)$, orthopyroxene $(15 \%)$, and olivine $(5 \%)$. Clinopyroxene occurs as large anhedral crystals up to $8 \mathrm{~mm}$ across; conspicuous exsolution of orthopyroxene lamellae and some alteration to serpentine and ironoxide minerals occur. Orthopyroxene and olivine crystals are smaller (1-2 mm). Olivine is completely pseudomorphed by serpentine and oxide minerals.

The serpentinite fragment (Sample 134-829A-44R-1, 1-4 cm) is coarse-grained, and is dominated by serpentine (65\%) with a subordinate amount of orthopyroxene (up to $4 \mathrm{~mm}$; around $25 \%$ ), clinopyroxene (up to $2 \mathrm{~mm}$; around 5\%) and anhedral $\mathrm{Cr}$-spinel (\%), which occupies interstitial spaces. No olivine relics have been preserved. Both pyroxenes contain exsolution lamellae. Some secondary tremolite crystals are also present.

\section{Site 830}

\section{Lavas}

The lavas, always recovered as clasts, vary in modal composition from moderately olivine + clinopyroxene-phyric basalts to highly plagioclase-phyric basalts, the latter type being the more common. Olivine phenocrysts are rare and always pseudomorphed by serpentine and minor calcite. Unaltered colorless clinopyroxene microphenocrysts $(0.2-2 \mathrm{~mm})$ often occur both as phenocryst, glomeroporphyritic aggregates, and in the groundmass. Idiomorphic plagioclase is the most common phase, being widely represented either as phenocryst (up to $2-3 \mathrm{~mm}$ in size) or in the groundmass. It is extensively altered to clay minerals, calcite and sericite and often has dark, isotropic melt inclusions 20 to $40 \mathrm{~mm}$ sized (up to $10 \%-15 \%$ of the crystal volume), 
Table 1. Major and trace element compositions of igneous rocks from Sites 829 and 830 .

\begin{tabular}{|c|c|c|c|c|c|c|c|c|c|c|c|c|c|c|c|}
\hline $\begin{array}{l}\text { Hole: } \\
\text { Core, section: }\end{array}$ & $\begin{array}{l}829 \mathrm{~A} \\
43 \mathrm{R}-3\end{array}$ & $\begin{array}{l}829 \mathrm{~A} \\
43 \mathrm{R}-3\end{array}$ & $\begin{array}{l}829 \mathrm{~A} \\
43 \mathrm{R}-3\end{array}$ & $\begin{array}{l}829 \mathrm{~A} \\
44 \mathrm{R}-1\end{array}$ & $\begin{array}{l}829 \mathrm{~A} \\
59 \mathrm{R}-1\end{array}$ & $\begin{array}{l}829 \mathrm{~A} \\
59 \mathrm{R}-1\end{array}$ & $\begin{array}{l}829 \mathrm{~A} \\
59 \mathrm{R}-1\end{array}$ & $\begin{array}{l}829 \mathrm{~A} \\
59 \mathrm{R}-1\end{array}$ & $\begin{array}{l}829 \mathrm{~A} \\
59 \mathrm{R}-1\end{array}$ & $\begin{array}{l}829 \mathrm{~A} \\
59 \mathrm{R}-1\end{array}$ & $\begin{array}{l}829 \mathrm{~A} \\
59 \mathrm{R}-1\end{array}$ & $\begin{array}{l}829 \mathrm{~A} \\
60 \mathrm{R}-1\end{array}$ & $\begin{array}{l}829 \mathrm{~A} \\
61 \mathrm{R}-1\end{array}$ & $\begin{array}{l}829 \mathrm{~A} \\
61 \mathrm{R}-1\end{array}$ & $\begin{array}{l}829 \mathrm{~A} \\
61 \mathrm{R}-1\end{array}$ \\
\hline $\begin{array}{l}\text { Inte, section: } \\
\text { Intal }\end{array}$ & $\begin{array}{l}43 R-3 \\
94-96\end{array}$ & $\begin{array}{l}43 k-3 \\
95-97\end{array}$ & $\begin{array}{c}43 R-3 \\
130-133\end{array}$ & $\begin{array}{c}44 R-1 \\
3-4\end{array}$ & $\begin{array}{l}59 R-1 \\
7-10\end{array}$ & $\begin{array}{l}59 \mathrm{R}-1 \\
45-50\end{array}$ & $\begin{array}{l}59 R-1 \\
81-85\end{array}$ & $\begin{array}{c}59 R-1 \\
105-108\end{array}$ & $\begin{array}{c}59 R-1 \\
118-120\end{array}$ & $\begin{array}{c}59 \mathrm{R}-1 \\
120-122\end{array}$ & $\begin{array}{c}59 R-1 \\
127-130\end{array}$ & $\begin{array}{c}60 R-1 \\
4-8\end{array}$ & $\begin{array}{c}6 \mid \mathrm{R}-1 \\
3-5\end{array}$ & $\begin{array}{l}61 \mathrm{R}-1 \\
30-33\end{array}$ & $\begin{array}{l}61 \mathrm{R}-1 \\
38-41\end{array}$ \\
\hline Depth (mbsf): & 403.1 & 403.2 & 403.5 & 407.4 & 533.0 & 533.4 & 533.7 & 534.0 & 534.1 & 534.1 & 534.2 & 542.6 & 552.3 & 552.6 & 552.7 \\
\hline \multirow[t]{2}{*}{ Rock type: } & Basalt & Basalt & Basalt & Serpentinite & Dolerite & Microgabbro & Dolerite & Microgabbro & Dolerite & Dolerite & Dolerite & Dolerite & Microgabbro & Microgabbro & Gabbro \\
\hline & $\mathbf{J}$ & $\mathrm{F}$ & $\mathrm{F}$ & F & F & J & F & J & $\mathrm{T}$ & F & $T$ & $\mathrm{~T}$ & J & $\mathrm{T}$ & $\mathrm{F}$ \\
\hline $\mathrm{SiO}_{2}$ & 50.86 & 49.83 & 49.51 & 45.89 & 47.21 & 48.86 & 47.10 & 48.04 & 47.91 & 47.29 & 47.95 & 48.39 & 48.07 & 47.63 & 46.29 \\
\hline $\mathrm{TiO}_{2}$ & 0.86 & 0.98 & 0.99 & 0.04 & 1.00 & 0.97 & 1.04 & 1.88 & 1.08 & 1.09 & 1.04 & 0.93 & 1.15 & 0.97 & 0.90 \\
\hline $\mathrm{Al}_{2} \mathrm{O}_{3}$ & 17.92 & 19.01 & 20.25 & 1.98 & 16.90 & 16.64 & 16.32 & 16.63 & 16.28 & 16.24 & 16.18 & 15.79 & 16.50 & 17.36 & 19.07 \\
\hline $\mathrm{Fe}_{2} \mathrm{O}_{3}$ & 1.39 & 1.34 & 1.21 & 1.06 & 1.36 & 1.34 & 1.38 & 1.68 & 1.43 & 1.39 & 1.46 & 1.49 & 1.54 & 1.66 & 1.40 \\
\hline $\mathrm{FeO}$ & 8.33 & 7.99 & 7.26 & 6.32 & 8.13 & 8.04 & 8.27 & 10.09 & 8.56 & 8.33 & 8.76 & 8.92 & 9.26 & 9.93 & 8.39 \\
\hline $\mathrm{MnO}$ & 0.17 & 0.13 & 0.11 & 0.15 & 0.17 & 0.18 & 0.17 & 0.20 & 0.19 & 0.17 & 0.20 & 0.33 & 0.22 & 0.17 & 0.15 \\
\hline $\mathrm{MgO}$ & 7.67 & 8.08 & 6.52 & 42.74 & 11.45 & 9.86 & 12.37 & 6.29 & 9.35 & 11.55 & 9.83 & 11.01 & 7.49 & 7.19 & 8.77 \\
\hline $\mathrm{CaO}$ & 9.15 & 8.54 & 10.41 & 1.61 & 11.36 & 11.97 & 10.86 & 10.61 & 12.36 & 11.48 & 11.92 & 8.71 & 12.94 & 11.86 & 12.07 \\
\hline $\mathrm{Na}_{2} \mathrm{O}$ & 2.38 & 2.98 & 3.08 & 0.17 & 2.21 & 2.03 & 2.27 & 4.25 & 2.68 & 2.26 & 2.52 & 2.32 & 2.75 & 3.04 & 2.80 \\
\hline $\mathrm{K}_{2} \mathrm{O}$ & 1.12 & 1.02 & 0.53 & 0.04 & 0.08 & 0.04 & 0.10 & 0.13 & 0.10 & 0.08 & 0.08 & 2.05 & 0.03 & 0.12 & 0.06 \\
\hline $\mathrm{P}_{2} \mathrm{O}_{5}$ & 0.15 & 0.11 & 0.11 & 0.00 & 0.11 & 0.06 & 0.11 & 0.18 & 0.07 & 0.11 & 0.07 & 0.06 & 0.05 & 0.07 & 0.11 \\
\hline LOI' & 4.37 & 12.37 & 11.46 & 19.76 & 1.44 & 2.68 & 2.19 & 3.93 & - & 2.32 & - & - & 1.05 & - & 1.14 \\
\hline $\mathrm{mgv}$ & 0.62 & 0.64 & 0.62 & 0.92 & 0.72 & 0.69 & 0.73 & 0.53 & 0.66 & 0.71 & 0.67 & 0.69 & 0.59 & 0.56 & 0.65 \\
\hline $\mathrm{Ni}$ & 43 & 29 & 33 & 2194 & 145 & 132 & 158 & 145 & 125 & 145 & 130 & 138 & 106 & 147 & 157 \\
\hline Co & & 36 & 34 & 135 & 52 & & 55 & & 54 & 51 & 55 & 49 & & 63 & 48 \\
\hline $\mathrm{Cr}$ & 78 & 88 & 77 & 3370 & 358 & 323 & 382 & 154 & 314 & 389 & 298 & 358 & 303 & 217 & 328 \\
\hline V & 244 & 235 & 267 & 95 & 294 & 286 & 305 & 269 & 273 & 312 & 256 & 276 & 288 & 196 & 247 \\
\hline $\mathrm{Rb}$ & 11 & 9 & 5 & n.d. & n.d. & n.d. & 2 & 3 & n.d. & n.d. & 1 & 11 & n.d. & 4 & 1 \\
\hline $\mathrm{Sr}$ & 248 & 232 & 232 & 23 & 69 & 63 & 67 & 124 & 70 & 64 & 68 & 68 & 88 & 104 & 101 \\
\hline $\mathrm{Ba}$ & 22 & 38 & 29 & 15 & 30 & 30 & 22 & 59 & 25 & 39 & 24 & 37 & 20 & 25 & 35 \\
\hline $\mathrm{Zr}$ & - & 34 & 40 & n.d. & 44 & 49 & 50 & 96 & 50 & 48 & 48 & 45 & 53 & 49 & 40 \\
\hline $\mathrm{Nb}$ & n.d. & 3 & n.d. & n.d. & 3 & 2 & 2 & 5 & 2 & 3 & 1 & 2 & 1 & 2 & 3 \\
\hline $\mathrm{La}$ & - & 3 & 2 & n.d. & 3 & - & 4 & - & - & 2 & - & - & - & - & n.d. \\
\hline $\mathrm{Ce}$ & 11 & 8 & 4 & n.d. & 8 & n.d. & 8 & n.d. & - & 5 & 4 & - & 11 & - & 3 \\
\hline \multirow[t]{2}{*}{$\mathrm{Y}$} & 31 & 22 & 25 & 2 & 28 & 27 & 31 & 40 & 30 & 32 & 29 & 26 & 33 & 32 & 29 \\
\hline & & & $\mathrm{T}$ & & & & & & & $\mathrm{N}$ & & $\mathrm{T}$ & & $\mathrm{T}$ & $\mathrm{N}$ \\
\hline $\mathrm{Y}$ & & & 21.1 & & & & & & & 26.6 & & 21.7 & & 27.3 & 22.9 \\
\hline $\mathrm{La}$ & & & 1.40 & & & & & & & 1.73 & & 1.00 & & 0.60 & 0.57 \\
\hline $\mathrm{Ce}$ & & & 5.80 & & & & & & & 4.81 & & 4.10 & & 3.10 & 3.30 \\
\hline $\mathrm{Nd}$ & & & 5.00 & & & & & & & 5.69 & & 3.30 & & 3.10 & 3.65 \\
\hline $\mathrm{Sm}$ & & & 2.35 & & & & & & & 2.79 & & 1.8 & & 2.21 & 2.13 \\
\hline Eu & & & 0.90 & & & & & & & 0.90 & & 0.69 & & 0.9 & 0. \\
\hline $\mathrm{Gd}$ & & & 3.22 & & & & & & & 3.75 & & 2.86 & & 3.6 & 2. \\
\hline & & & 3.55 & & & & & & & 4.21 & & 37 & & 4.8 & 3.69 \\
\hline $\mathrm{Er}$ & & & 2.28 & & & & & & & 2.46 & & 25 & & 3. & 2 \\
\hline $\mathrm{Yb}$ & & & 218 & & & & & & & 260 & & 2.66 & & 312 & 219 \\
\hline $\mathrm{Lu}$ & & & 0.33 & & & & & & & 0.39 & & 0.40 & & 0.46 & 0.31 \\
\hline $\mathrm{Ti} / \mathrm{Zr}$ & & 172 & 149 & & 136 & 120 & 125 & 117 & 129 & 136 & 130 & 124 & 130 & 119 & 134 \\
\hline $\mathrm{Ba} / \mathrm{Nb}$ & & 12 & & & 9 & 16 & 9 & 13 & 13 & 11 & 24 & 19 & 20 & 13 & 11 \\
\hline $\mathrm{Zr} / \mathrm{Nb}$ & & 11 & & & 13 & 26 & 22 & 20 & 25 & 14 & 48 & 23 & 53 & 25 & 13 \\
\hline
\end{tabular}

Notes: Analyses conducted aboard the JOIDES Resolution (J), at Ferrara University (F), Tohoku University (T), and at CRPG (N [Nancy, France]). Y analyses carried out by ICP are reported withdecimals. $\mathrm{mgv}=\mathrm{Mg} / \mathrm{Mg}+\mathrm{Fe}^{2+}(\mathrm{mol} \%) ; \mathrm{LOI}=$ loss on ignition. $-=$ not determined; n.d. $=$ not detected. 
Table 1 (continued).

\begin{tabular}{|c|c|c|c|c|c|c|c|c|c|c|c|c|c|c|c|c|}
\hline Hole: & $\begin{array}{l}829 \mathrm{~A} \\
61 \mathrm{R}-1\end{array}$ & $829 \mathrm{~A}$ & $829 \mathrm{~A}$ & $829 \mathrm{~A}$ & $829 \mathrm{~A}$ & $829 \mathrm{~A}$ & $830 \mathrm{~B}$ & $830 \mathrm{~B}$ & $830 \mathrm{~B}$ & $830 \mathrm{~B}$ & $830 \mathrm{~B}$ & $830 \mathrm{~B}$ & $830 \mathrm{C}$ & $830 \mathrm{C}$ & & \\
\hline Core, section: & $61 \mathrm{R}-1$ & $61 \mathrm{R}-1$ & $61 \mathrm{R}-1$ & $61 \mathrm{R}-1$ & $62 \mathrm{R}-1$ & $64 \mathrm{R}-1$ & $14 \mathrm{R}-1$ & $14 \mathrm{R}-1$ & $14 \mathrm{R}-1$ & $22 \mathrm{R}-1$ & $22 \mathrm{R}-1$ & $22 \mathrm{R}-1$ & $2 \mathrm{R}-1$ & $3 \mathrm{R}-1$ & & \\
\hline Interval $(\mathrm{cm})$ : & $38-41$ & $56-59$ & $71-74$ & $80-86$ & $49-52$ & $10-13$ & $13-17$ & $13-17$ & $59-64$ & $32-33$ & $34-37$ & $40-44$ & $6-8$ & $3-4$ & AVG 1 & BR \\
\hline Depth (mbsf): & 552.7 & 552.9 & 553.0 & 553.1 & 562.3 & 581.3 & 175.0 & 175.0 & 175.5 & 252.7 & 252.7 & 252.8 & 244.5 & 254.5 & & \\
\hline Rock type: & Gabbro & Microgabbro & $\begin{array}{c}\text { Gabbro } \\
\text { F }\end{array}$ & $\underset{\mathrm{J}}{\text { Microgabbro }}$ & $\begin{array}{c}\text { Gabbro } \\
\text { F }\end{array}$ & $\underset{\mathrm{J}}{\text { Gabbro }}$ & $\begin{array}{c}\text { Basalt } \\
\text { F }\end{array}$ & Basalt & $\begin{array}{l}\text { Basalt } \\
\mathrm{T}\end{array}$ & $\begin{array}{l}\text { Basalt } \\
\mathrm{J}\end{array}$ & $\begin{array}{l}\text { Basalt } \\
\text { F }\end{array}$ & $\begin{array}{l}\text { Basalt } \\
\mathrm{T}\end{array}$ & $\begin{array}{c}\text { Basalt } \\
\mathrm{J}\end{array}$ & $\begin{array}{c}\text { Basalt } \\
\mathrm{J}\end{array}$ & $\begin{array}{l}\text { Andesite } \\
\text { F }\end{array}$ & $\begin{array}{c}\text { Basalt } \\
\text { F }\end{array}$ \\
\hline $\mathrm{SiO}_{2}$ & & 47.90 & 46.71 & 47.99 & 47.18 & 49.37 & 47.68 & & 49.85 & 49.98 & 48.48 & 50.79 & 51.27 & 51.45 & 59.13 & 37.91 \\
\hline $\mathrm{TiO}_{2}^{2}$ & & 0.97 & 0.81 & 0.92 & 1.03 & 0.74 & 0.78 & & 0.70 & 1.34 & 1.48 & 1.38 & 1.49 & 1.19 & 1.09 & 2.62 \\
\hline $\mathrm{Al}_{2} \mathrm{O}_{3}$ & & 17.52 & 18.48 & 17.94 & 20.67 & 17.55 & 17.37 & & 13.94 & 18.48 & 18.94 & 17.29 & 18.68 & 16.28 & 17.59 & 11.48 \\
\hline $\mathrm{Fe}_{2} \mathrm{O}_{3}$ & & 1.50 & 1.38 & 1.46 & 1.18 & 1.27 & 1.42 & & 1.58 & 1.40 & 1.45 & 1.49 & 1.36 & 1.44 & 6.64 & 12.26 \\
\hline $\mathrm{FeO}$ & & 9.00 & 8.28 & 8.79 & 7.08 & 7.59 & 8.50 & & 9.49 & 8.42 & 8.73 & 8.92 & 8.15 & 8.66 & & 12.20 \\
\hline $\mathrm{MnO}$ & & 0.22 & 0.15 & 0.16 & 0.13 & 0.18 & 0.19 & & 0.31 & 0.21 & 0.20 & 0.22 & 0.24 & 0.31 & 0.10 & 0.17 \\
\hline $\mathrm{MgO}$ & & 8.45 & 8.88 & 8.61 & 6.88 & 6.87 & 10.35 & & 10.03 & 4.64 & 5.13 & 4.78 & 5.24 & 6.76 & 1.18 & 13.57 \\
\hline $\mathrm{CaO}$ & & 12.06 & 12.39 & 10.61 & 12.52 & 14.00 & 10.01 & & 10.52 & 11.63 & 11.62 & 10.55 & 9.53 & 10.98 & 4.87 & 13.30 \\
\hline $\mathrm{Na}_{2} \mathrm{O}$ & & 2.29 & 2.74 & 3.39 & 3.10 & 2.33 & 2.99 & & 2.16 & 3.03 & 3.03 & 3.45 & 3.35 & 2.25 & 4.26 & 3.15 \\
\hline $\mathrm{K}_{2} \mathrm{O}$ & & 0.05 & 0.07 & 0.07 & 0.10 & 0.06 & 0.59 & & 1.30 & 0.60 & 0.66 & 0.85 & 0.42 & 0.45 & 2.83 & 1.39 \\
\hline $\mathrm{P}_{2} \mathrm{O}_{5}$ & & 0.05 & 0.10 & 0.06 & 0.12 & 0.03 & 0.13 & & 0.11 & 0.26 & 0.26 & 0.28 & 0.27 & 0.22 & 0.48 & 1.31 \\
\hline Lor' & & 3.21 & 1.85 & 4.62 & 2.85 & 4.00 & 3.56 & & 6.12 & 4.41 & - & 5.07 & 3.51 & 7.07 & - & - \\
\hline $\mathrm{mgv}$ & & 0.63 & 0.66 & 0.64 & 0.63 & 0.62 & 0.68 & & 0.65 & 0.50 & 0.51 & 0.49 & 0.53 & 0.58 & & \\
\hline $\mathrm{Ni}$ & & 189 & 191 & 202 & 212 & 177 & 86 & & 51 & 30 & 28 & 24 & 25 & 50 & 14 & 263 \\
\hline Co & & 80 & 53 & - & 54 & 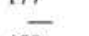 & 44 & & 45 & 0 & 31 & 31 & - & - & 15 & 54 \\
\hline $\mathrm{Cr}$ & & 395 & 439 & 336 & 331 & 450 & 290 & & 266 & 25 & 52 & 51 & 8 & 113 & 14 & 340 \\
\hline $\mathrm{v}$ & & 229 & 223 & 205 & 235 & 212 & 381 & & 321 & 281 & 307 & n.d. & $238^{\circ}$ & 310 & 120 & 239 \\
\hline $\mathrm{Rb}$ & & 1 & 2 & 1 & 1 & n.d. & 6 & & 16 & 7 & 9 & 14 & 5 & 7 & 70 & 50 \\
\hline $\mathrm{Sr}$ & & 114 & 98 & 126 & 111 & 94 & 499 & & 210 & 361 & 403 & 384 & 244 & 347 & 672 & 1382 \\
\hline $\mathrm{Ba}$ & & 25 & 25 & 22 & 28 & 24 & 122 & & 195 & 184 & 213 & 351 & 274 & 153 & 1198 & 1200 \\
\hline $\mathrm{Zr}$ & & 51 & 33 & 49 & 40 & 41 & 42 & & 37 & 141 & 98 & 92 & 169 & 112 & 225 & 230 \\
\hline $\mathrm{Nb}$ & & i & n.d. & 1 & 4 & 1 & 1 & & 2 & 6 & 5 & 5 & 4 & 3 & 16 & 105 \\
\hline $\mathrm{La}$ & & - & n.d. & - & 3 & - & 7 & & - & - & 19 & - & - & - & 39 & 90 \\
\hline $\mathrm{Ce}$ & & 7 & 5 & 3 & 7 & 1 & 17 & & 13 & 25 & 38 & - & 17 & 17 & 70 & 161 \\
\hline \multirow[t]{2}{*}{$\mathrm{Y}$} & & 30 & 27 & 28 & 32 & 26 & 14 & & 16 & 27 & 28 & - & 38 & 24 & 20 & 28 \\
\hline & $\mathrm{T}$ & & & & & & $\mathrm{N}$ & $\mathrm{T}$ & $\mathrm{T}$ & & & $\mathrm{T}$ & & & & \\
\hline $\mathrm{Y}$ & 23.7 & & & & & & 12.9 & - & 13.2 & & & 25.0 & & & & \\
\hline $\mathrm{La}$ & 0.30 & & & & & & 4.74 & 4.30 & 5.30 & & & 11.2 & & & & \\
\hline $\mathrm{Ce}$ & 2.60 & & & & & & 12.1 & 10.7 & 11.1 & & & 27.2 & & & & \\
\hline $\mathrm{Nd}$ & 2.60 & & & & & & 7.62 & 6.50 & 6.60 & & & 17.2 & & & & \\
\hline $\mathrm{Sm}$ & 1.82 & & & & & & 2.29 & 2.09 & 2.11 & & & 5.13 & & & & \\
\hline $\mathrm{Eu}$ & 0.80 & & & & & & 0.70 & 0.67 & 0.63 & & & 1.41 & & & & \\
\hline $\mathrm{Gd}$ & 3.33 & & & & & & 2.30 & 2.41 & 2.29 & & & 4.57 & & & & \\
\hline Dy & 4.10 & & & & & & 2.35 & 2.37 & 2.31 & & & 4.78 & & & & \\
\hline $\mathrm{Er}$ & 2.68 & & & & & & 1.19 & 1.50 & 1.56 & & & 2.82 & & & & \\
\hline $\mathrm{Yb}$ & 2.57 & & & & & & 1.32 & $\begin{array}{l}1.46 \\
1.46\end{array}$ & $\begin{array}{l}1.50 \\
1.54\end{array}$ & & & 2.59 & & & & \\
\hline Lu & 0.40 & & & & & & 0.19 & 0.24 & 0.25 & & & 0.40 & & & & \\
\hline $\mathrm{Ti} / \mathrm{Zr}$ & & 113 & 147 & 112 & 156 & 108 & 111 & & 113 & 57 & 91 & 90 & 53 & 64 & & \\
\hline $\mathrm{Ba} / \mathrm{Nb}$ & & 36 & & 20 & 7 & 35 & 135 & & 98 & 31 & 44 & 69 & 69 & 51 & & \\
\hline $\mathrm{Zr} / \mathrm{Nb}$ & & 73 & & 45 & 9 & 58 & 47 & & 19 & 24 & 20 & 18 & 42 & 37 & & \\
\hline
\end{tabular}


rounded or lobate, that are completely replaced by chlorite, and usually oriented along crystallographic directions, or zonally arranged.

The groundmass is hypocrystalline, intergranular to hyalopilitic, with microlites of plagioclase, small grains of subequant clinopyroxene and tiny elongated rods of ilmenite set in an altered, devitrified glassy mesostasis, sometimes with a spherulitic texture. Vesicles, which are filled with chlorite, calcite and zeolites, can amount to as much as $20 \mathrm{vol} \%$.

\section{Volcanic Breccias}

These breccias are made up of subangular rock fragments and single crystals set in a microcrystalline to mainly devitrified glassy matrix. The composition and texture of rock fragments are similar to those of the lavas, consisting of variably altered basalts ranging from aphyric to highly plagioclase-phyric. Although the compositional variation of the volcanic clasts is wider than that described for the lavas of the first group, the relative mineral abundances and textural relationships are very similar. Fragments are rounded to subrounded and sometimes show gradual, diffuse transitions into the groundmass. Some fragments are lobate or irregular in shape and partially engulf isolated crystals. Two clasts of fine-grained gabbro were also found in Section 134-830C-3R-1.

Single crystals mainly comprise large idiomorphic, fractured, twinned and strongly zoned clinopyroxenes (up to $3 \mathrm{~mm}$ ), and plagioclase (up to $1.5 \mathrm{~mm}$ ), with subordinate olivine, amphibole, and small anhedral oxides. They appear to be similar in composition to phases in the associated lava fragments and in the clasts of the previous group.

\section{MINERAL CHEMISTRY}

\section{Site 827}

Representative analyses of clinopyroxenes and amphiboles from an andesitic clast are reported in Tables 2 and 3. Clinopyroxene plot in the diopside-augite field of the Wo-En-Fs diagram (Fig. 2B), with $\mathrm{mgv}\left(\mathrm{mgv}=\mathrm{Mg} /\left[\mathrm{Mg}+\mathrm{Fe}^{2+}\right]=0.95-0.80\right)$. They have a low Ti content with respect to $\mathrm{Al}^{\mathrm{IV}}$, consistent with clinopyroxene phenocrysts in lavas from intraoceanic volcanic arcs (Fig. 3).

Anorthite content in plagioclase shows a large variation $\left(\mathrm{An}_{48-65}\right.$ in one sample and $\mathrm{An}_{60-94}$ in another).

Titanium magnesio-hastingsite is fairly abundant and richer in $\mathrm{FeO}$ than clinopyroxene (mgv from 0.64 to 0.66 ; Table 3 ).

\section{Site 829}

\section{Clinopyroxene}

This mineral is less common than plagioclase, but it plays a major role in the chemical identification of the parental magma (Leterrier et al., 1982; Beccaluva et al., 1989). Representative analyses are reported in Table 2, and compositions are plotted in the Di-Hd-En-Fs quadrilateral (Figs. 2A and B, and 4). As for plagioclase, clinopyroxene in the basalts has a narrower compositional range than in the gabbros. In the basalts, they fall in the endiopside-augite fields (with a few points also plotting in the salite field) and vary from $W_{35} \mathrm{En}_{55} \mathrm{Fs}_{10}$ to $\mathrm{Wo}_{47} \mathrm{En}_{36} \mathrm{Fs}_{17}$. Clinopyroxene rims in the dolerite (Sample 134-829A$59 \mathrm{R}-1,81-86 \mathrm{~cm}$ ) have the highest iron content, with $\mathrm{Wo}_{41} \mathrm{En}_{34} \mathrm{Fs}_{25}$. In the gabbros, an almost complete compositional range of clinopyroxenes fractionating from a tholeiitic magma is recorded (Fig. 4). Although the whole rock geochemistry does not show a very high $\mathrm{FeO}$ and $\mathrm{TiO}_{2}$ contents (see Table 1), clinopyroxene rims almost reach pure hedenbergite composition (Sample 134-829A-61R-1, 20-22 cm), passing from augite through ferro-augite. This extreme iron enrichment may indicate crystallization in a nearly-closed system, in which the most evolved interstitial liquid could not be interchanged with fresh, undifferentiated magma. In Figure 4, clinopyroxene compositions from gabbros recovered from mid-ocean ridges and from high-Ti ophiolitic complexes are also drawn (Hebert et al., 1989). As expected, the two fields delimit very similar areas, with clinopyroxenes in ophiolitic gabbros having a higher Fe content. The majority of analyses from Site 829 gabbros plot inside these fields, although some analyses have even higher total $\mathrm{FeO}$ contents than those from high-Ti ophiolitic complex. The similarity between clinopyroxene compositions from

Table 2. Representative clinopyroxene compositions from andesitic, basaltic, gabbroic, and ultramafic rocks from Sites 827 and 829.

\begin{tabular}{|c|c|c|c|c|c|c|c|c|c|c|c|c|c|c|c|c|}
\hline \multirow[t]{2}{*}{$\begin{array}{l}\text { Hole: } \\
\text { Core, section: } \\
\text { Interval }(\mathrm{cm}) \text { : } \\
\text { Rock type: }\end{array}$} & & $\begin{array}{c}827 \mathrm{~B} \\
\text { 18R-CC } \\
0-2 \\
\text { Andesite }\end{array}$ & & & & $\begin{array}{l}829 \mathrm{~A} \\
43 \mathrm{R}-3 \\
94-95 \\
\text { Basalt }\end{array}$ & & & & $\begin{array}{c}829 \mathrm{~A} \\
59 \mathrm{R}-1 \\
3-4 \\
\text { Basalt }\end{array}$ & & & & $\begin{array}{c}829 \mathrm{~A} \\
44 \mathrm{R}-1 \\
1-2 \\
\text { erpentinit }\end{array}$ & & \\
\hline & A ph c & A phr & $\mathrm{mph}$ & $\mathrm{Aph}$ & B ph & $\mathrm{C}$ ph & $\mathrm{gm}$ & $\mathrm{gm}$ & $\mathrm{ph}$ & $\mathrm{gm}$ & $\mathrm{gm}$ & $\mathrm{Ac}$ & $\mathrm{Ar}$ & $\mathrm{B} \mathrm{c}$ & $\mathrm{Br}$ & C \\
\hline $\begin{array}{l}\mathrm{SiO}_{2} \\
\mathrm{TiO}_{2} \\
\mathrm{Al}_{2} \mathrm{O}_{3} \\
\mathrm{Fe}_{2} \mathrm{O}_{3} \\
\mathrm{FeO} \\
\mathrm{MnO} \\
\mathrm{MgO} \\
\mathrm{CaO} \\
\mathrm{Na}_{2} \mathrm{O} \\
\mathrm{Cr}_{2} \mathrm{O}_{3} \\
\text { Total }\end{array}$ & $\begin{array}{r}52.77 \\
0.23 \\
2.01 \\
2.49 \\
1.74 \\
0.05 \\
17.00 \\
23.64 \\
0.20 \\
0.29 \\
100.13\end{array}$ & $\begin{array}{r}49.43 \\
1.03 \\
4.36 \\
3.62 \\
6.14 \\
0.45 \\
14.21 \\
20.75 \\
0.33 \\
0.00 \\
100.32\end{array}$ & $\begin{array}{r}52.15 \\
0.37 \\
1.87 \\
2.62 \\
6.48 \\
0.41 \\
15.44 \\
20.96 \\
0.31 \\
0.00 \\
100.61\end{array}$ & $\begin{array}{r}53.74 \\
0.16 \\
1.66 \\
0.00 \\
6.07 \\
0.22 \\
19.84 \\
17.15 \\
0.12 \\
0.24 \\
98.95\end{array}$ & $\begin{array}{r}47.19 \\
1.25 \\
7.47 \\
2.10 \\
7.36 \\
0.23 \\
12.97 \\
20.21 \\
0.20 \\
0.02 \\
98.99\end{array}$ & $\begin{array}{r}51.92 \\
0.41 \\
2.94 \\
0.61 \\
6.91 \\
0.12 \\
15.71 \\
20.74 \\
0.18 \\
0.16 \\
99.54\end{array}$ & $\begin{array}{r}52.91 \\
0.30 \\
2.83 \\
0.00 \\
5.98 \\
0.12 \\
17.22 \\
20.25 \\
0.15 \\
0.21 \\
99.76\end{array}$ & $\begin{array}{r}47.65 \\
1.00 \\
7.56 \\
0.29 \\
8.48 \\
0.24 \\
13.40 \\
18.67 \\
0.29 \\
0.00 \\
97.59\end{array}$ & $\begin{array}{r}48.41 \\
1.42 \\
5.13 \\
2.70 \\
7.78 \\
0.14 \\
12.05 \\
22.09 \\
0.32 \\
0.46 \\
100.04\end{array}$ & $\begin{array}{r}47.28 \\
1.77 \\
6.32 \\
3.05 \\
7.25 \\
0.09 \\
11.55 \\
22.42 \\
0.32 \\
0.24 \\
100.05\end{array}$ & $\begin{array}{r}47.95 \\
1.33 \\
5.71 \\
3.26 \\
6.61 \\
0.28 \\
11.59 \\
22.83 \\
0.37 \\
0.18 \\
99.92\end{array}$ & $\begin{array}{r}52.32 \\
0.13 \\
3.44 \\
0.37 \\
2.15 \\
0.08 \\
16.48 \\
23.24 \\
0.28 \\
1.44 \\
98.49\end{array}$ & $\begin{array}{r}51.98 \\
0.25 \\
3.09 \\
0.73 \\
1.65 \\
0.14 \\
16.59 \\
23.33 \\
0.25 \\
1.35 \\
98.00\end{array}$ & $\begin{array}{r}53.74 \\
0.18 \\
2.67 \\
0.00 \\
2.32 \\
0.14 \\
17.07 \\
23.81 \\
0.22 \\
1.14 \\
100.15\end{array}$ & $\begin{array}{r}53.13 \\
0.24 \\
3.16 \\
0.21 \\
2.42 \\
0.04 \\
17.00 \\
23.15 \\
0.29 \\
1.47 \\
99.64\end{array}$ & $\begin{array}{r}53.30 \\
0.20 \\
2.95 \\
0.00 \\
2.49 \\
0.13 \\
16.82 \\
23.36 \\
0.24 \\
1.21 \\
99.48\end{array}$ \\
\hline $\begin{array}{l}\mathrm{Si} \\
\mathrm{Ti} \\
\mathrm{Al}^{\mathrm{y}} \\
\mathrm{Al} \\
\mathrm{Fe}^{3+} \\
\mathrm{Fe}^{2+} \\
\mathrm{Mn} \\
\mathrm{Mg} \\
\mathrm{Ca} \\
\mathrm{Na} \\
\mathrm{Cr} \\
\text { Total }\end{array}$ & $\begin{array}{l}1.915 \\
0.006 \\
0.085 \\
0.089 \\
0.069 \\
0.054 \\
0.002 \\
0.929 \\
0.918 \\
0.015 \\
0.009 \\
4.082\end{array}$ & $\begin{array}{l}1.830 \\
0.029 \\
0.170 \\
0.197 \\
0.102 \\
0.192 \\
0.014 \\
0.792 \\
0.822 \\
0.025 \\
0.000 \\
4.173\end{array}$ & $\begin{array}{l}1.918 \\
0.010 \\
0.082 \\
0.084 \\
0.074 \\
0.202 \\
0.013 \\
0.855 \\
0.825 \\
0.023 \\
0.000 \\
4.086\end{array}$ & $\begin{array}{l}1.963 \\
0.004 \\
0.037 \\
0.071 \\
0.000 \\
0.185 \\
0.007 \\
1.080 \\
0.671 \\
0.009 \\
0.007 \\
4.028\end{array}$ & $\begin{array}{l}1.776 \\
0.035 \\
0.224 \\
0.332 \\
0.060 \\
0.232 \\
0.008 \\
0.728 \\
0.815 \\
0.015 \\
0.001 \\
4.223\end{array}$ & $\begin{array}{l}1.920 \\
0.011 \\
0.080 \\
0.128 \\
0.017 \\
0.214 \\
0.004 \\
0.866 \\
0.822 \\
0.013 \\
0.005 \\
4.075\end{array}$ & $\begin{array}{l}1.934 \\
0.008 \\
0.066 \\
0.122 \\
0.000 \\
0.183 \\
0.004 \\
0.938 \\
0.793 \\
0.011 \\
0.006 \\
4.059\end{array}$ & $\begin{array}{l}1.809 \\
0.029 \\
0.191 \\
0.338 \\
0.008 \\
0.269 \\
0.008 \\
0.758 \\
0.760 \\
0.021 \\
0.000 \\
4.191\end{array}$ & $\begin{array}{l}1.813 \\
0.040 \\
0.187 \\
0.227 \\
0.076 \\
0.244 \\
0.004 \\
0.673 \\
0.886 \\
0.023 \\
0.014 \\
4.173\end{array}$ & $\begin{array}{l}1.775 \\
0.050 \\
0.225 \\
0.280 \\
0.086 \\
0.228 \\
0.003 \\
0.466 \\
0.902 \\
0.023 \\
0.007 \\
4.038\end{array}$ & $\begin{array}{l}1.801 \\
0.037 \\
0.199 \\
0.253 \\
0.092 \\
0.208 \\
0.009 \\
0.649 \\
0.919 \\
0.027 \\
0.005 \\
4.194\end{array}$ & $\begin{array}{l}1.907 \\
0.004 \\
0.093 \\
0.148 \\
0.010 \\
0.066 \\
0.002 \\
0.895 \\
0.908 \\
0.020 \\
0.041 \\
4.052\end{array}$ & $\begin{array}{l}1.906 \\
0.007 \\
0.094 \\
0.134 \\
0.020 \\
0.051 \\
0.004 \\
0.906 \\
0.916 \\
0.017 \\
0.039 \\
4.055\end{array}$ & $\begin{array}{l}1.930 \\
0.005 \\
0.070 \\
0.113 \\
0.000 \\
0.070 \\
0.004 \\
0.914 \\
0.916 \\
0.015 \\
0.033 \\
4.037\end{array}$ & $\begin{array}{l}1.913 \\
0.007 \\
0.087 \\
0.134 \\
0.006 \\
0.073 \\
0.001 \\
0.912 \\
0.893 \\
0.020 \\
0.042 \\
4.045\end{array}$ & $\begin{array}{l}1.926 \\
0.006 \\
0.074 \\
0.126 \\
0.000 \\
0.075 \\
0.004 \\
0.906 \\
0.904 \\
0.017 \\
0.035 \\
4.037\end{array}$ \\
\hline mgv & 0.95 & 0.80 & 0.81 & 0.85 & 0.76 & 0.80 & 0.84 & 0.74 & 0.73 & 0.67 & 0.76 & 0.93 & 0.95 & 0.93 & 0.93 & 0.92 \\
\hline $\begin{array}{l}\text { Wo } \\
\text { En } \\
\text { Fs }\end{array}$ & $\begin{array}{r}46.6 \\
47.1 \\
6.3\end{array}$ & $\begin{array}{l}42.8 \\
41.2 \\
16.0\end{array}$ & $\begin{array}{l}41.9 \\
43.4 \\
14.7\end{array}$ & $\begin{array}{r}34.5 \\
55.6 \\
9.9\end{array}$ & $\begin{array}{l}44.3 \\
39.5 \\
16.2\end{array}$ & $\begin{array}{l}42.7 \\
45.1 \\
12.2\end{array}$ & $\begin{array}{r}41.4 \\
48.9 \\
9.7\end{array}$ & $\begin{array}{l}42.1 \\
42.0 \\
15.8\end{array}$ & $\begin{array}{l}47.1 \\
35.7 \\
17.2\end{array}$ & $\begin{array}{l}53.5 \\
27.7 \\
18.8\end{array}$ & $\begin{array}{l}49.0 \\
34.6 \\
16.5\end{array}$ & $\begin{array}{r}48.3 \\
47.6 \\
4.2\end{array}$ & $\begin{array}{r}48.3 \\
47.8 \\
4.0\end{array}$ & $\begin{array}{r}48.1 \\
48.0 \\
3.9\end{array}$ & $\begin{array}{r}47.4 \\
48.4 \\
4.2\end{array}$ & $\begin{array}{r}47.9 \\
47.9 \\
4.2\end{array}$ \\
\hline
\end{tabular}

Notes: $\mathrm{mgv}=\mathrm{Mg} / \mathrm{Mg} \mathrm{Fe}{ }^{2}$ (at $\%$; Wo, En, and Fs indicate wollastonite, enstatite, and ferrosilite percentages. ph = phenocrysts; $\mathrm{mph}=$ microphenocryst; gm = groundmass; $\mathrm{c}=$ core; $\mathrm{r}$ $=\operatorname{rim} . \mathrm{A}, \mathrm{B}$, and $\mathrm{C}$ indicate different crystals. 


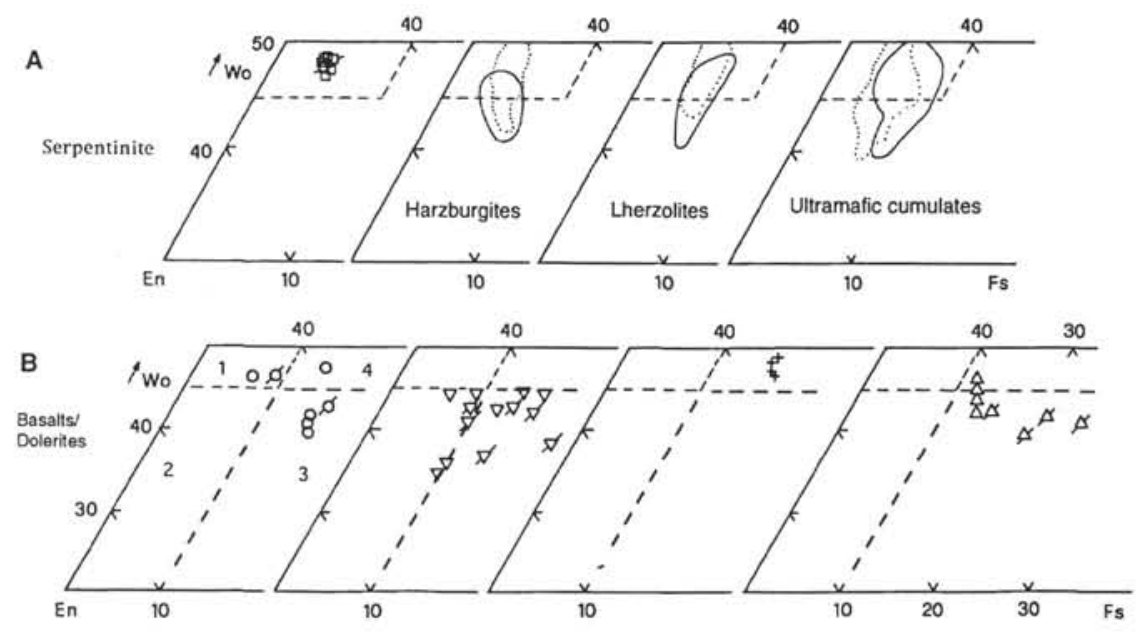

Hole $827 \mathrm{C}$

Gore Rim

○ o 18RCC, $0.2 \mathrm{~cm}$
Hole 829A

$$
\begin{aligned}
& \text { Core Rim Core Rim } \\
& \nabla \gg 43 R-3,94-95 \mathrm{~cm}+59 \mathrm{R}-1,3-4 \mathrm{~cm} \\
& \text { 마 44R-1, 1-2 cm } \Delta \text { ه } 59 \mathrm{R}-1,81-86 \mathrm{~cm}
\end{aligned}
$$

Figure 2. Clinopyroxene compositions of igneous rocks recovered from Sites 827 and 829 in the Wo-En-Fs diagram. 1, 2, 3, and 4 indicate diopside, endiopside, augite, and salite compositions. A. Clinopyroxenes in serpentinite. B. Clinopyroxenes in andesite (Site 827), basalts, and dolerites (Site 829). Fields of lherzolites, harzburgites, and ultramafic cumulates from major ocean basins (dotted line) and from a high-Ti ophiolitic complex (Northern Apennine; solid line) are from Hebert et al. (1989).

\begin{tabular}{|c|c|c|c|c|c|c|c|c|c|c|c|c|c|c|c|c|}
\hline \multirow[t]{2}{*}{$\begin{array}{l}\text { Hole: } \\
\text { Core, section: } \\
\text { Interval }(\mathrm{cm}) \text { : } \\
\text { Rock type: }\end{array}$} & & $\begin{array}{c}82 \\
591 \\
81 \\
\text { Dol }\end{array}$ & $\begin{array}{l}\text { A } \\
-1 \\
86 \\
\text { rite }\end{array}$ & & & $\begin{array}{r}82 \\
61 R \\
1- \\
\text { Gab }\end{array}$ & $\begin{array}{l}9 A \\
2-1 \\
3 \\
\text { bro }\end{array}$ & & & $\begin{array}{r}829 \\
61 \mathrm{R} \\
20- \\
\text { Microg }\end{array}$ & $\begin{array}{l}A \\
1 \\
2 \\
\text { abbro }\end{array}$ & & & $\begin{array}{r}829 \\
61 \mathrm{R} \\
36- \\
\text { Microg }\end{array}$ & $\begin{array}{l}\text { A } \\
\text { R-1 } \\
38 \\
\text { gabbro }\end{array}$ & \\
\hline & A ph c & $\mathrm{Aphr}$ & B ph c & B ph $\mathrm{r}$ & $\mathrm{Ac}$ & $\mathrm{Ar}$ & B c & $\mathrm{B} \mathrm{r}$ & $\mathrm{Ac}$ & Ar & $\mathrm{B} \mathrm{c}$ & $\mathrm{B} \mathrm{r}$ & A c & A r & $\mathrm{B} \mathrm{c}$ & $\mathrm{Br}$ \\
\hline $\begin{array}{l}\mathrm{SiO}_{2} \\
\mathrm{TiO}_{2} \\
\mathrm{Al}_{2} \mathrm{O}_{3} \\
\mathrm{Fe}_{2} \mathrm{O}_{3} \\
\mathrm{FeO} \\
\mathrm{MnO} \\
\mathrm{MgO} \\
\mathrm{CaO} \\
\mathrm{Na}_{2} \mathrm{O} \\
\mathrm{Cr}_{2} \mathrm{O}_{3} \\
\text { Total }\end{array}$ & $\begin{array}{r}50.48 \\
0.81 \\
4.26 \\
1.21 \\
6.13 \\
0.17 \\
14.58 \\
21.54 \\
0.26 \\
0.52 \\
99.46\end{array}$ & $\begin{array}{r}50.02 \\
0.98 \\
1.98 \\
1.49 \\
13.41 \\
0.38 \\
11.51 \\
19.43 \\
0.32 \\
0.00 \\
99.52\end{array}$ & $\begin{array}{r}50.62 \\
0.85 \\
3.36 \\
2.03 \\
7.06 \\
0.29 \\
14.66 \\
20.58 \\
0.31 \\
0.08 \\
99.76\end{array}$ & $\begin{array}{r}50.23 \\
0.96 \\
2.41 \\
0.82 \\
13.81 \\
0.49 \\
11.42 \\
19.20 \\
0.36 \\
0.00 \\
99.70\end{array}$ & $\begin{array}{r}48.31 \\
0.16 \\
1.28 \\
5.34 \\
19.35 \\
0.78 \\
3.85 \\
20.75 \\
0.93 \\
0.05 \\
100.75\end{array}$ & $\begin{array}{r}47.82 \\
0.17 \\
1.02 \\
5.31 \\
20.79 \\
0.83 \\
2.59 \\
20.63 \\
1.00 \\
0.00 \\
100.16\end{array}$ & $\begin{array}{r}48.77 \\
0.47 \\
1.30 \\
4.28 \\
18.84 \\
0.69 \\
5.20 \\
20.50 \\
0.79 \\
0.00 \\
100.84\end{array}$ & $\begin{array}{r}48.27 \\
0.17 \\
0.96 \\
5.19 \\
21.22 \\
0.78 \\
2.63 \\
20.80 \\
0.98 \\
0.04 \\
101.00\end{array}$ & $\begin{array}{r}50.90 \\
0.76 \\
1.38 \\
1.77 \\
12.40 \\
0.49 \\
11.34 \\
20.68 \\
0.42 \\
0.00 \\
100.14\end{array}$ & $\begin{array}{r}49.36 \\
0.37 \\
1.19 \\
2.35 \\
18.62 \\
0.63 \\
6.31 \\
20.49 \\
0.56 \\
0.00 \\
99.89\end{array}$ & $\begin{array}{r}49.55 \\
1.25 \\
2.96 \\
2.98 \\
9.50 \\
0.24 \\
12.03 \\
20.94 \\
0.51 \\
0.00 \\
99.96\end{array}$ & $\begin{array}{r}49.59 \\
0.96 \\
1.61 \\
2.44 \\
14.89 \\
0.48 \\
8.34 \\
21.35 \\
0.55 \\
0.07 \\
100.29\end{array}$ & $\begin{array}{r}51.41 \\
0.65 \\
3.17 \\
2.26 \\
5.31 \\
0.16 \\
14.89 \\
22.25 \\
0.33 \\
0.27 \\
100.43\end{array}$ & $\begin{array}{r}50.92 \\
0.87 \\
1.46 \\
2.63 \\
11.54 \\
0.34 \\
11.34 \\
21.24 \\
0.51 \\
0.02 \\
100.85\end{array}$ & $\begin{array}{r}50.42 \\
0.78 \\
4.20 \\
2.88 \\
5.13 \\
0.12 \\
14.74 \\
21.60 \\
0.39 \\
0.23 \\
100.25\end{array}$ & $\begin{array}{r}50.56 \\
0.56 \\
1.28 \\
2.72 \\
13.87 \\
0.45 \\
9.84 \\
20.93 \\
0.53 \\
0.00 \\
100.73\end{array}$ \\
\hline $\begin{array}{l}\mathrm{Si} \\
\mathrm{Ti}^{\mathrm{i}} \\
\mathrm{Al}^{\mathrm{IV}} \\
\mathrm{Al}^{\mathrm{I}} \\
\mathrm{Fe}^{3+} \\
\mathrm{Fe}^{2+} \\
\mathrm{Mn} \\
\mathrm{Mg} \\
\mathrm{Ca} \\
\mathrm{Na} \\
\mathrm{Cr} \\
\text { Total }\end{array}$ & $\begin{array}{l}1.869 \\
0.023 \\
0.131 \\
0.186 \\
0.034 \\
0.190 \\
0.005 \\
0.805 \\
0.855 \\
0.019 \\
0.015 \\
4.116\end{array}$ & $\begin{array}{l}1.918 \\
0.028 \\
0.083 \\
0.090 \\
0.043 \\
0.430 \\
0.012 \\
0.658 \\
0.798 \\
0.024 \\
0.000 \\
4.082\end{array}$ & $\begin{array}{l}1.884 \\
0.024 \\
0.116 \\
0.147 \\
0.057 \\
0.220 \\
0.009 \\
0.814 \\
0.821 \\
0.022 \\
0.002 \\
4.113\end{array}$ & $\begin{array}{l}1.920 \\
0.028 \\
0.080 \\
0.109 \\
0.024 \\
0.441 \\
0.016 \\
0.651 \\
0.786 \\
0.027 \\
0.000 \\
4.080\end{array}$ & $\begin{array}{l}1.920 \\
0.005 \\
0.080 \\
0.060 \\
0.160 \\
0.643 \\
0.026 \\
0.228 \\
0.884 \\
0.072 \\
0.002 \\
4.078\end{array}$ & $\begin{array}{l}1.929 \\
0.005 \\
0.071 \\
0.049 \\
0.161 \\
0.701 \\
0.028 \\
0.156 \\
0.892 \\
0.078 \\
0.000 \\
4.071\end{array}$ & $\begin{array}{l}1.923 \\
0.014 \\
0.077 \\
0.061 \\
0.127 \\
0.621 \\
0.023 \\
0.305 \\
0.866 \\
0.061 \\
0.000 \\
4.077\end{array}$ & $\begin{array}{l}1.931 \\
0.005 \\
0.069 \\
0.045 \\
0.156 \\
0.710 \\
0.026 \\
0.157 \\
0.892 \\
0.076 \\
0.001 \\
4.067\end{array}$ & $\begin{array}{l}1.938 \\
0.022 \\
0.063 \\
0.062 \\
0.051 \\
0.395 \\
0.016 \\
0.643 \\
0.844 \\
0.031 \\
0.000 \\
4.063\end{array}$ & $\begin{array}{l}1.948 \\
0.011 \\
0.052 \\
0.055 \\
0.070 \\
0.615 \\
0.021 \\
0.371 \\
0.866 \\
0.043 \\
0.000 \\
4.052\end{array}$ & $\begin{array}{l}1.875 \\
0.036 \\
0.125 \\
0.132 \\
0.085 \\
0.301 \\
0.008 \\
0.678 \\
0.849 \\
0.038 \\
0.000 \\
4.125\end{array}$ & $\begin{array}{l}1.920 \\
0.028 \\
0.080 \\
0.074 \\
0.071 \\
0.482 \\
0.016 \\
0.481 \\
0.886 \\
0.042 \\
0.002 \\
4.079\end{array}$ & $\begin{array}{l}1.890 \\
0.018 \\
0.110 \\
0.138 \\
0.063 \\
0.163 \\
0.005 \\
0.816 \\
0.876 \\
0.024 \\
0.008 \\
4.102\end{array}$ & $\begin{array}{l}1.924 \\
0.025 \\
0.076 \\
0.065 \\
0.075 \\
0.365 \\
0.011 \\
0.639 \\
0.860 \\
0.037 \\
0.001 \\
4.076\end{array}$ & $\begin{array}{l}1.858 \\
0.022 \\
0.142 \\
0.183 \\
0.080 \\
0.158 \\
0.004 \\
0.810 \\
0.853 \\
0.028 \\
0.007 \\
4.136\end{array}$ & $\begin{array}{l}1.936 \\
0.016 \\
0.064 \\
0.058 \\
0.079 \\
0.438 \\
0.015 \\
0.561 \\
0.859 \\
0.040 \\
0.000 \\
4.065\end{array}$ \\
\hline $\mathrm{mgv}$ & 0.81 & 0.60 & 0.79 & 0.60 & 0.26 & 0.18 & 0.33 & 0.18 & 0.62 & 0.38 & 0.69 & 0.50 & 0.83 & 0.64 & 0.84 & 0.56 \\
\hline $\begin{array}{l}\text { Wo } \\
\text { En } \\
\text { Fs }\end{array}$ & $\begin{array}{l}45.3 \\
42.6 \\
12.1\end{array}$ & $\begin{array}{l}41.1 \\
33.9 \\
25.0\end{array}$ & $\begin{array}{l}42.7 \\
42.4 \\
14.9\end{array}$ & $\begin{array}{l}41.0 \\
33.9 \\
25.1\end{array}$ & $\begin{array}{l}45.5 \\
11.7 \\
42.7\end{array}$ & $\begin{array}{r}46.0 \\
8.0 \\
46.0\end{array}$ & $\begin{array}{l}44.6 \\
15.7 \\
39.7\end{array}$ & $\begin{array}{r}45.9 \\
8.1 \\
46.0\end{array}$ & $\begin{array}{l}43.3 \\
33.0 \\
23.7\end{array}$ & $\begin{array}{l}44.6 \\
19.1 \\
36.3\end{array}$ & $\begin{array}{l}44.2 \\
35.3 \\
20.5\end{array}$ & $\begin{array}{l}45.8 \\
24.8 \\
29.4\end{array}$ & $\begin{array}{l}45.6 \\
42.4 \\
12.0\end{array}$ & $\begin{array}{l}44.1 \\
32.8 \\
23.1\end{array}$ & $\begin{array}{l}44.8 \\
42.5 \\
12.7\end{array}$ & $\begin{array}{l}44.0 \\
28.8 \\
27.2\end{array}$ \\
\hline
\end{tabular}
Compositions of microphenocrysts and groundmass crystals are indicated as core and rim, respectively.

Table 2 (continued). 

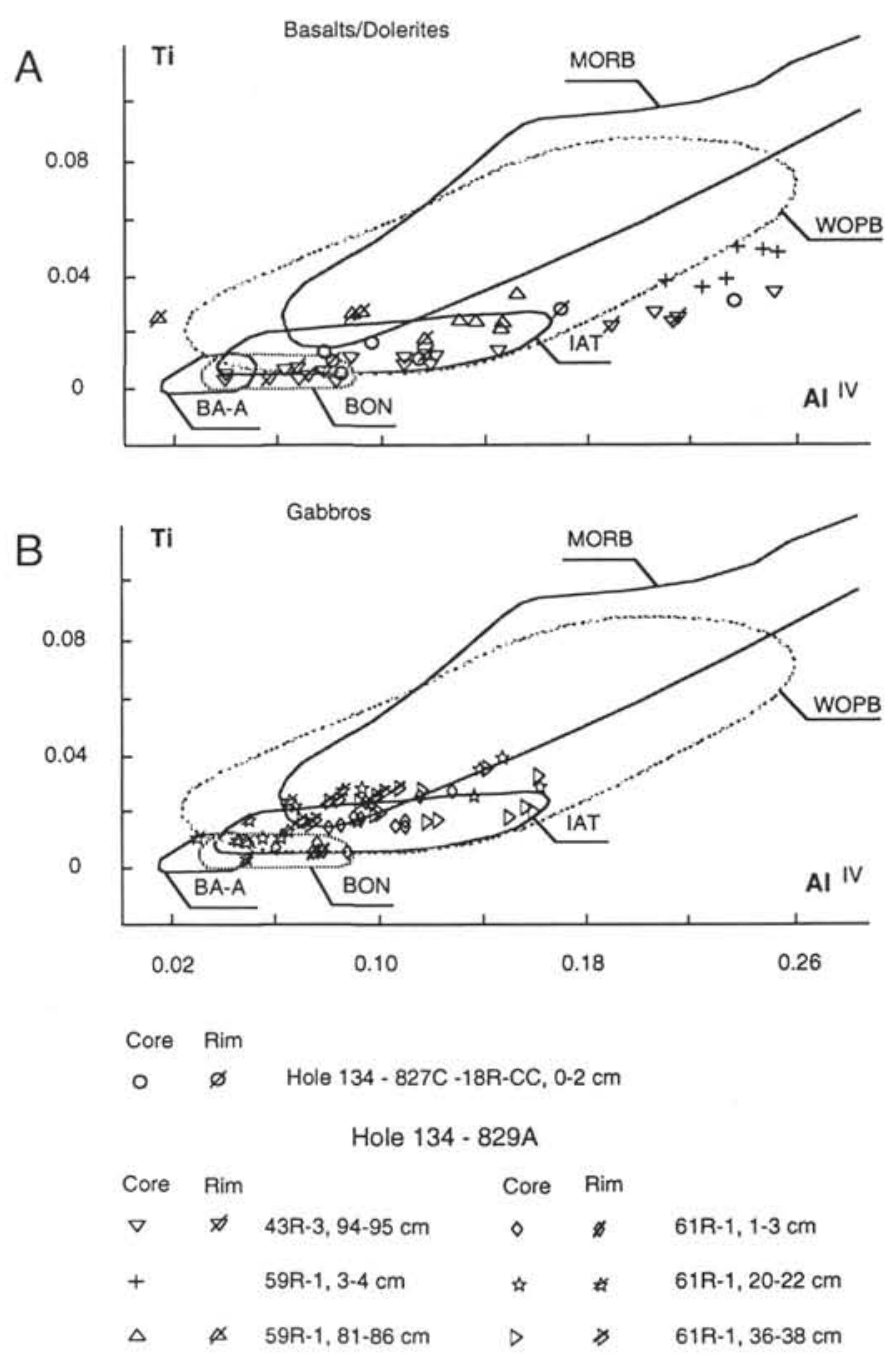

Figure 3. $\mathrm{Ti}$ vs. $\mathrm{Al}^{\mathrm{IV}}$ contents (at\%) in clinopyroxenes from (A) basaltic and (B) gabbroic rocks of Hole 829A. Fields of clinopyroxenes from mid-ocean ridge basalts (MORB), within-oceanic plate basalts (WOPB), island arc tholeiites (IAT), boninites (BON), and basaltic andesites, and andesites (BA-A) from the forearc region are from Beccaluva et al. (1989). Compositions of microphenocrysts and groundmass crystals are indicated as core and rim, respectively.

gabbros of Site 829 and those from oceanic basins is also stressed by the $\mathrm{Ti}$ vs. Cr diagram (Fig. 5). However, in the $\mathrm{Ti}$ vs. $\mathrm{Al}^{\mathrm{IV}}$ diagram, in which compositional fields from different tectonic settings are reported (Fig. 3; Beccaluva et al., 1989), analyses of clinopyroxene of basalts and gabbros from Site 829 plot inside the island arc tholeiite (IAT) field, overlapping the lowermost part of MORB field. On average, they show significantly lower Ti contents compared to clinopyroxene from major ocean basin gabbros, with clinopyroxene from effusive rocks having even lower $\mathrm{Ti}$ contents than those from the gabbros.

Clinopyroxenes in the serpentinite clast are plotted in Figure 2A. They fall in a very restricted area, well within the field of suboceanic mantle tectonite peridotites, but close to the field for $\mathrm{Mg}$-rich clinopyroxenes from oceanic ultramafic cumulates (Serri et al., 1985; Hebert et al., 1989). Similarly, $\mathrm{Ti}$ and $\mathrm{Cr}$ contents of these clinopyroxene preclude effective separation between oceanic tectonite peridotites and ultramafic cumulates (Fig. 5; Hebert et al., 1989).

\section{Plagioclase}

Plagioclase is the most common mineral, in basalts and gabbros. Representative compositions are reported in Table 4 and are plotted

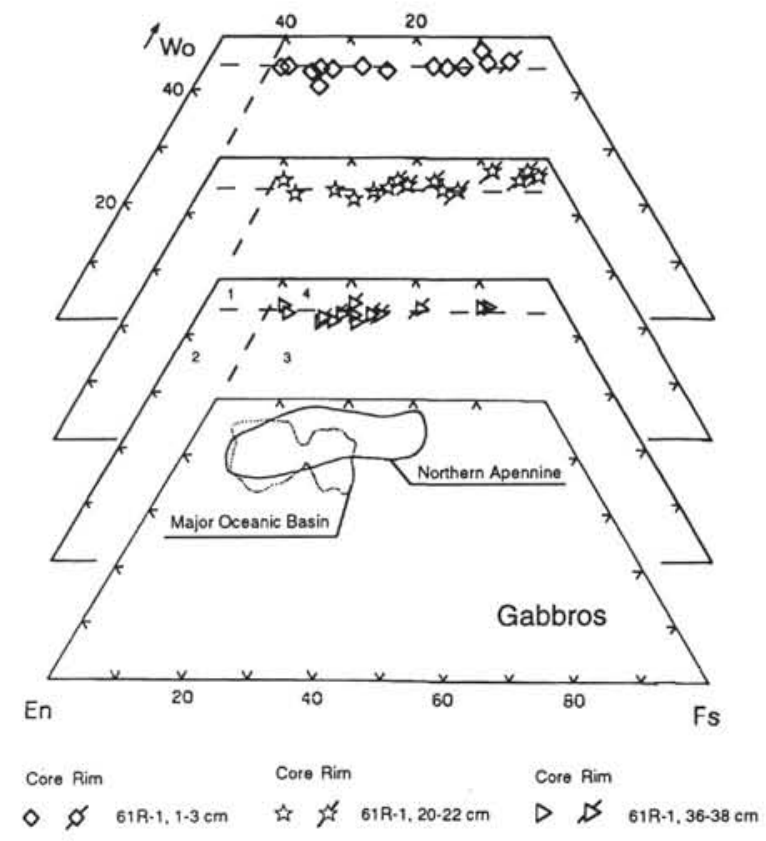

Figure 4. Clinopyroxene compositions of gabbros and microgabbros from Site 829. Fields of gabbros from major ocean basins and from a high-Ti ophiolitic complex (Northern Apennine) are from Hebert et al. (1989). Composition of microphenocrysts and groundmass crystals are indicated as core and rim, respectively.

on $\mathrm{An}$-Ab-Or diagrams in Figure 6. They range from $\mathrm{An}_{78.8}$ to $\mathrm{An}_{22.6}$. In basaltic rocks, An contents show a more restricted range $\left(\mathrm{An}_{76.9-62.3}\right.$ in microphenocrysts and phenocryst cores; $\mathrm{An}_{54.3}$ in phenocryst rims; Fig. 6) than for plagioclase in the gabbros $\left(\mathrm{An}_{78,0-55.2}\right.$ and down to $\mathrm{An}_{22.6}$; Fig. 6), the latter suggesting equilibrium with an evolved interstitial liquid.

\section{Olivine}

Olivine has only been analyzed in the gabbros from the base of Hole 829A. It is strongly zoned, from $\mathrm{Fo}_{82-62}$ in crystal cores to $\mathrm{Fo}_{68-30}$ in rims (Table 3). NiO contents vary between $0.29-0.0 \mathrm{wt} \%$, and $0.11-0.0 \mathrm{wt} \%$ in cores and rims respectively. These olivine rim compositions provide further evidence for late stage crystallization from evolved, iron-rich liquid, as recognized also for plagioclase and clinopyroxene.

\section{Oxides}

Representative analyses of magnetite, Ti-magnetite, and ilmenite from basalts and gabbros are reported in Table 4. A few $\mathrm{Cr}$-spinels in the serpentinite have also been analyzed.

In basalts and dolerites the $\mathrm{TiO}_{2}$ contents of the magnetite ranges between 3.09 and $14.02 \mathrm{wt} \%$, with ulvospinel and jacobsite molecules in the range $9.31 \%-42.81 \%$ and $0.84 \%-2.35 \%$, respectively; magnetite in the gabbros has higher $\mathrm{TiO}_{2}$ and $\mathrm{MnO}$ contents. In fact, $\mathrm{TiO}_{2}$ contents in gabbros cluster in the range 14.6-21.3 wt\% (apart from two analyses with $\sim 2 \mathrm{wt} \%$ ), and $\mathrm{MnO}$ is always higher than 0.55 wt\%. Acicular ilmenite analyzed in Sample 134-829A-59R-1, 3-4 $\mathrm{cm}$, has an anomalously high $\mathrm{MnO}$ content (up to $15 \mathrm{wt} \%$ ).

The $\mathrm{Cr} /(\mathrm{Cr}+\mathrm{Al})$ vs. $\mathrm{Mg} /\left(\mathrm{Mg}+\mathrm{Fe}^{2+}\right)$ values of spinels in the ultramafic clast (Sample 134-829A-44R-1, 3-4 cm) are plotted in Figure 7. Their position in this diagram is consistent with an harzburgitic bulk composition but, as for clinopyroxenes, they also overlap into field for oceanic cumulates. 
Table 3. Representative amphibole and olivine compositions from basaltic and gabbroic rocks from Sites 827 and 829.

\begin{tabular}{|c|c|c|c|c|c|c|c|c|c|c|c|c|c|c|c|c|}
\hline \multirow[t]{2}{*}{$\begin{array}{l}\text { Hole: } \\
\text { Core, section: } \\
\text { Interval }(\mathrm{cm}) \text { : } \\
\text { Rock type: }\end{array}$} & . & $\begin{array}{c}827 \mathrm{~B} \\
18 \mathrm{R}-\mathrm{CC} \\
0-2 \\
\text { Basalt }\end{array}$ & & & $\begin{array}{l}29 A \\
R-1 \\
-3 \\
\text { bboro }\end{array}$ & $\begin{array}{l}\text { Hole: } \\
\text { Core, section: } \\
\text { Interval (cm): } \\
\text { Rock type: }\end{array}$ & & & $\begin{array}{l}29 \mathrm{~A} \\
\text { IR-1 } \\
1-3 \\
\text { abbro }\end{array}$ & & & & $\begin{array}{r}8 \\
61 \\
3 \\
\text { Micr } \\
\end{array}$ & $\begin{array}{l}29 \mathrm{~A} \\
1 \mathrm{R}-1 \\
6-38 \\
\text { ogabbro }\end{array}$ & & \\
\hline & amph & amph & amph & amph & amph & & A ol c & $\mathrm{A} \mathrm{ol} \mathrm{r}$ & r B ol c & C $\mathrm{B} \mathrm{ol} \mathrm{r}$ & A ol c & c A ol $\mathrm{r}$ & B ol c & c $\mathrm{B} \mathrm{ol} \mathrm{r}$ & C ol c & c C ol r \\
\hline $\mathrm{SiO}_{2}$ & 43.14 & 39.98 & 42.37 & 43.97 & 36.15 & $\mathrm{SiO}_{2}$ & 38.35 & 33.39 & 39.14 & 34.80 & 39.57 & 35.96 & 39.06 & 35.77 & 39.44 & 37.31 \\
\hline $\mathrm{TiO}_{2}^{2}$ & 3.03 & 2.41 & 3.47 & 1.02 & 0.22 & $\mathrm{TiO}_{2}$ & 0.00 & 0.05 & 0.00 & 0.03 & 0.02 & 0.11 & 0.04 & 0.03 & 0.01 & 0.00 \\
\hline $\mathrm{Al}_{2} \mathrm{O}_{3}$ & 11.06 & 15.28 & 11.96 & 7.43 & 14.01 & $\mathrm{Al}_{2} \mathrm{O}_{3}$ & 0.02 & 0.02 & 0.05 & 0.04 & 0.07 & 0.03 & 0.05 & 0.03 & 0.04 & 0.03 \\
\hline $\mathrm{Fe}_{2} \mathrm{O}_{3}$ & 0.00 & 0.00 & 0.00 & 0.00 & 0.00 & $\mathrm{FeO}$ & 25.56 & 50.26 & 22.57 & 43.09 & 19.10 & 36.63 & 18.97 & 37.01 & 17.75 & 29.68 \\
\hline $\mathrm{FeO}$ & 12.84 & 12.29 & 13.41 & 22.25 & 28.88 & $\mathrm{MnO}$ & 0.47 & 1.08 & 0.43 & 0.76 & 0.29 & 0.69 & 0.33 & 0.72 & 0.37 & 0.44 \\
\hline $\mathrm{MnO}$ & 0.36 & 0.17 & 0.17 & 0.54 & 0.89 & $\mathrm{MgO}$ & 37.22 & 16.21 & 39.57 & 22.63 & 42.21 & 26.63 & 41.74 & 27.03 & 42.72 & 33.87 \\
\hline $\mathrm{MgO}$ & 13.84 & 13.22 & 13.50 & 9.83 & 1.96 & $\mathrm{CaO}$ & 0.42 & 0.56 & 0.43 & 0.55 & 0.38 & 0.49 & 0.36 & 0.57 & 0.44 & 0.39 \\
\hline $\mathrm{CaO}$ & 10.75 & 11.82 & 10.57 & 7.94 & 10.41 & $\mathrm{NiO}$ & 0.07 & 0.00 & 0.14 & 0.00 & 0.11 & 0.06 & 0.01 & 0.04 & 0.19 & 0.00 \\
\hline $\mathrm{Na}_{2} \mathrm{O}$ & 2.49 & 2.57 & 2.67 & 4.48 & 3.64 & Total & 102.111 & 101.561 & 102.321 & 101.90 & 101.74 & 100.601 & 100.56 & 101.20 & 100.95 & 101.73 \\
\hline $\mathrm{K}_{2} \mathrm{O}$ & 0.26 & 0.37 & 0.28 & 0.00 & 0.00 & & & & & & & & & & & \\
\hline $\mathrm{Cr}_{2} \mathrm{O}_{3}$ & 0.03 & 0.02 & 0.08 & 0.04 & 0.00 & $\mathrm{Si}$ & 0.993 & 0.992 & 0.996 & 0.989 & 0.996 & 1.001 & 0.995 & 0.993 & 0.996 & 0.990 \\
\hline $\mathrm{H}_{2} \mathrm{O}$ & 2.04 & 2.04 & 2.04 & - & - & $\mathrm{Ti}$ & 0.000 & 0.001 & 0.000 & 0.001 & 0.000 & 0.002 & 0.001 & 0.001 & 0.000 & 0.000 \\
\hline Total & 99.841 & 100.171 & 100.52 & 97.51 & 96.15 & $\mathrm{Al}$ & 0.001 & 0.001 & 0.001 & 0.001 & 0.002 & 0.001 & 0.002 & 0.001 & 0.001 & 0.001 \\
\hline & & & & & & $\mathrm{Fe}$ & 0.554 & 1.249 & 0.480 & 1.024 & 0.402 & 0.853 & 0.404 & 0.859 & 0.375 & 0.659 \\
\hline $\mathrm{Si}$ & 6.354 & 5.889 & 6.217 & 6.735 & 5.851 & Mn & 0.010 & 0.027 & 0.009 & 0.018 & 0.006 & 0.016 & 0.007 & 0.017 & 0.008 & 0.010 \\
\hline $\mathrm{Ti}$ & 0.336 & 0.267 & 0.382 & 0.118 & 0.026 & $\mathrm{Mg}$ & 1.436 & 0.718 & 1.501 & 0.959 & 1.584 & 1.105 & 1.585 & 1.118 & 1.608 & 1.339 \\
\hline $\mathrm{Al}_{3}$ & 1.919 & 2.652 & 2.068 & 1.342 & 2.672 & $\mathrm{Ca}$ & 0.012 & 0.018 & 0.012 & 0.017 & 0.010 & 0.015 & 0.010 & 0.017 & 0.012 & 0.011 \\
\hline $\mathrm{Fe}^{3+}$ & 0.000 & 0.000 & 0.000 & 0.000 & 0.000 & $\mathrm{Ni}$ & 0.001 & 0.000 & 0.003 & 0.000 & 0.002 & 0.001 & 0.000 & 0.001 & 0.004 & 0.000 \\
\hline $\mathrm{Fe}$ & 1.581 & 1.514 & 1.646 & 2.851 & 3.909 & Total & 3.01 & 3.01 & 3.00 & 3.01 & 3.00 & 3.00 & 3.00 & 3.01 & 3.00 & 3.01 \\
\hline $\mathrm{Mn}$ & 0.044 & 0.021 & 0.022 & 0.070 & 0.122 & & & & & & & & & & & \\
\hline $\mathrm{Mg}$ & 3.037 & 2.903 & 2.953 & 2.244 & 0.472 & Fo & 0.72 & 036 & 0.76 & 0.48 & 0.80 & 0.56 & 0.80 & 0.57 & 0.81 & 0.67 \\
\hline $\mathrm{Ca}$ & 1.696 & 1.866 & 1.661 & 1.304 & 1.806 & $\mathrm{Fa}$ & 0.28 & 0.64 & 0.24 & 0.52 & 0.20 & 0.44 & 0.20 & 0.43 & 0.19 & 0.33 \\
\hline $\mathrm{Na}$ & 0.710 & 0.735 & 0.758 & 1.331 & 1.142 & & & & & & & & & & & \\
\hline K & 0.049 & 0.070 & 0.052 & 0.000 & 0.000 & & & & & & & & & & & \\
\hline $\mathrm{Cr}$ & 0.000 & 0.003 & 0.009 & 0.005 & 0.000 & & & & & & & & & & & \\
\hline Total & 15.726 & 15.917 & 15.759 & 15.994 & 16.000 & & & & & & & & & & & \\
\hline mgv & 0.66 & 0.66 & 0.64 & 0.44 & 0.11 & & & & & & & & & & & \\
\hline
\end{tabular}

Notes: $\mathrm{Fo}$ and $\mathrm{Fa}$ indicate forsterite and fayalite percentages. amph $=$ amphibole; ol $=$ olivine. $-=$ not determined. Other abbreviations as in Table 2 .

\section{WHOLE ROCK GEOCHEMISTRY}

Major, minor, and trace element (including REE) analyses of samples recovered from Sites 829 and 830 are reported in Table 1, and are discussed below with emphasis on determining the magmatic affinities and tectonic setting of eruption of these lavas and associated intrusive igneous rocks.

\section{Site 829}

Samples from this site show different degrees of alteration, reflected in variable LOI values (in the range of 1.05-4.62 wt\%) and also evident in the frequent appearance of normative nepheline, particularly for the gabbroic rocks (up to $5.4 \mathrm{wt} \%$ ). Two samples of sparsely clinopyroxene + plagioclase-phyric basalts have a very high LOI contents (11.46-12.37), resulting from the high percentage of filled vesicles in this lithotype. As expected, the ultramafic clast has the highest LOI content (19.8 wt\%).

Basaltic rocks from this site are quite primitive (mgv: 0.64-0.62), whereas dolerites and gabbros have a wider range of $\mathrm{mgv}(0.73-0.53)$. Enrichment in $\mathrm{FeO}$ and $\mathrm{TiO}_{2}$, as indicated by clinopyroxene and olivine compositions, is evident in Sample 134-829A-59R-1, 105$108 \mathrm{~cm}$, which has the lowest $\mathrm{MgO}$ coupled with the highest $\mathrm{FeO}$ and $\mathrm{TiO}_{2}$ contents. Two basalts from this site have $\mathrm{Ti} / \mathrm{Zr}$ values of 149 and 172 (Fig. 8), values more typical of IAT than normal MORB. In the MORB-normalized incompatible element diagram, these samples are enriched in low field strength elements (LFSE) (by a factor of three for $\mathrm{Sr}$ and seven for $\mathrm{Ba}$, relative to MORB) and slightly depleted in high field strength elements (HFSE), particularly Zr (Fig. 9A). Although significant alteration of Site 829 basaltic rocks may have modified their original LFSE contents, their overall geochemical features are unlike those of MORB, and taken together with the HFSE contents transitional between typical MORB and IAT compositions (Fig. 9A), suggest a weak subduction-related signature for the mantle source of these magmas (Pearce, 1983).

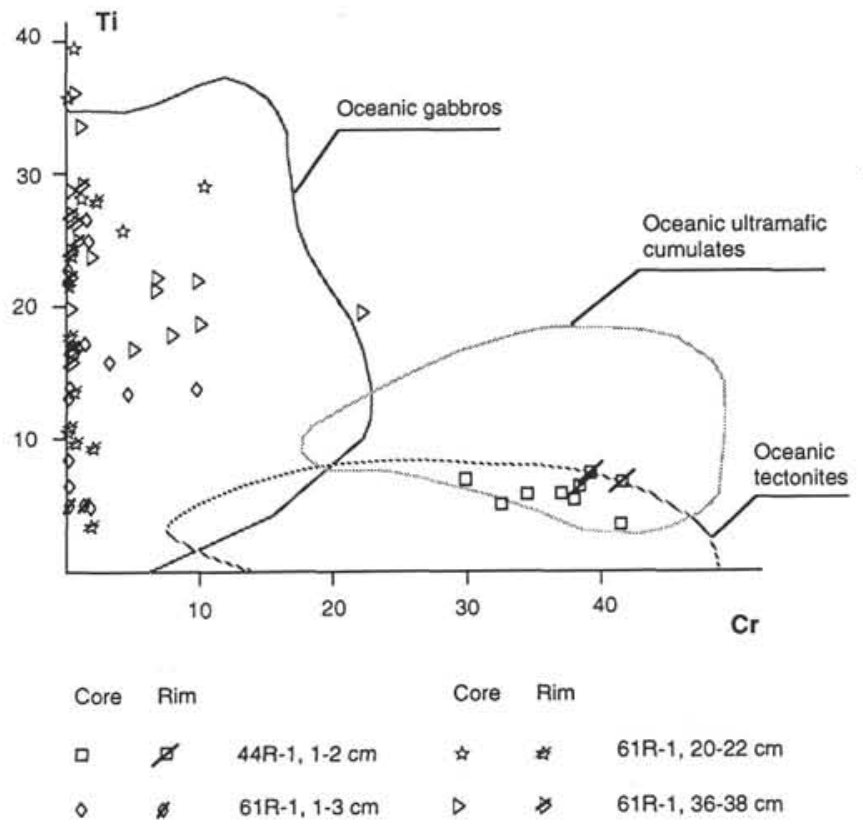

Figure 5. Ti vs. $\mathrm{Cr}(\mathrm{at} \% \times 1000)$ contents in clinopyroxenes from serpentinite (Sample 134-829A-44R-1, 1-2 cm) and gabbros from Hole 829A. Fields of oceanic tectonites, ultramafic cumulates, and gabbros are from Hebert et al. (1989).

Gabbros generally represent cumulate mineral assemblages with variable proportions of intercumulus phases. On the $\mathrm{Ti}-\mathrm{Zr}$ diagram (Fig. 8) they define a field that broadly overlaps with that of the basalts. Dolerites have $\mathrm{Ti} / \mathrm{Zr}$ values that vary from 124 to 136 (avg. 130), and for the gabbros and microgabbros, the range is 108-156 (avg. 126); all are slightly higher than average values for normal MORB (103-109; 
Table 4. Representative plagioclase and oxide compositions from basaltic, gabbroic, and ultramafic rocks recovered from Site 829 .

\begin{tabular}{|c|c|c|c|c|c|c|c|c|}
\hline \multirow[t]{2}{*}{$\begin{array}{l}\text { Hole: } \\
\text { Core, section: } \\
\text { Interval }(\mathrm{cm}) \text { : } \\
\text { Rock type: } \\
\text { * }\end{array}$} & $\begin{array}{r}82 \\
431 \\
94 \\
\mathrm{Ba} \\
\end{array}$ & $\begin{array}{l}9 \mathrm{~A} \\
\mathrm{R}-3 \\
-95 \\
\text { salt } \\
\end{array}$ & $\begin{array}{r}82 \\
591 \\
3 \\
\mathrm{Ba} \\
\end{array}$ & $\begin{array}{l}9 \mathrm{~A} \\
\mathrm{R}-1 \\
-4 \\
\text { salt } \\
\end{array}$ & & $\begin{array}{l}829 \mathrm{~A} \\
9 \mathrm{R}-1 \\
1-86 \\
\text { olerite }\end{array}$ & & $\begin{array}{l}29 \mathrm{~A} \\
1 \mathrm{R}-1 \\
1-3 \\
\text { abbro }\end{array}$ \\
\hline & A pl gm & B pl gm & B pl ph c & B pl ph r & $\mathrm{plc}$ & $\mathrm{pl} \mathrm{r}$ & $\mathrm{plc}$ & $\mathrm{pl} \mathrm{r}$ \\
\hline $\begin{array}{l}\mathrm{SiO}_{2} \\
\mathrm{Al}_{2} \mathrm{O}_{3} \\
\mathrm{Fe}_{2} \mathrm{O}_{3} \\
\mathrm{MgO}_{3} \\
\mathrm{CaO} \\
\mathrm{Na}_{2} \mathrm{O} \\
\mathrm{K}_{2} \mathrm{O} \\
\mathrm{Tot}\end{array}$ & $\begin{array}{r}53.02 \\
28.25 \\
1.35 \\
0.00 \\
13.17 \\
4.00 \\
0.05 \\
99.84\end{array}$ & $\begin{array}{r}51.30 \\
30.09 \\
0.96 \\
0.00 \\
15.09 \\
3.31 \\
0.04 \\
100.80\end{array}$ & $\begin{array}{r}51.25 \\
30.36 \\
0.70 \\
0.33 \\
15.09 \\
3.15 \\
0.00 \\
100.88\end{array}$ & $\begin{array}{r}55.15 \\
27.03 \\
1.06 \\
0.17 \\
11.28 \\
5.16 \\
0.14 \\
99.99\end{array}$ & $\begin{array}{r}48.43 \\
31.98 \\
0.45 \\
0.23 \\
16.43 \\
2.56 \\
0.02 \\
100.11\end{array}$ & $\begin{array}{r}62.09 \\
23.54 \\
0.61 \\
0.00 \\
5.77 \\
8.76 \\
0.14 \\
100.92\end{array}$ & $\begin{array}{r}48.37 \\
32.03 \\
0.46 \\
0.00 \\
16.56 \\
2.71 \\
0.06 \\
100.18\end{array}$ & $\begin{array}{r}62.13 \\
23.31 \\
0.36 \\
0.00 \\
5.10 \\
9.58 \\
0.13 \\
100.60\end{array}$ \\
\hline $\begin{array}{l}\mathrm{Si} \\
\mathrm{Al} \\
\mathrm{Fe} \\
\mathrm{Mg} \\
\mathrm{Ca} \\
\mathrm{Na} \\
\mathrm{K} \\
\mathrm{Tot}\end{array}$ & $\begin{array}{l}2.417 \\
1.517 \\
0.046 \\
0.000 \\
0.643 \\
0.354 \\
0.003 \\
4.980\end{array}$ & $\begin{array}{l}2.328 \\
1.609 \\
0.033 \\
0.000 \\
0.734 \\
0.292 \\
0.003 \\
4.998\end{array}$ & $\begin{array}{l}2.323 \\
1.622 \\
0.027 \\
0.023 \\
0.733 \\
0.277 \\
0.000 \\
5.004\end{array}$ & $\begin{array}{l}2.501 \\
1.445 \\
0.040 \\
0.012 \\
0.548 \\
0.454 \\
0.008 \\
5.007\end{array}$ & $\begin{array}{l}2.222 \\
1.730 \\
0.016 \\
0.016 \\
0.808 \\
0.228 \\
0.001 \\
5.020\end{array}$ & $\begin{array}{l}2.740 \\
1.225 \\
0.020 \\
0.000 \\
0.273 \\
0.750 \\
0.008 \\
5.016\end{array}$ & $\begin{array}{l}2.220 \\
1.733 \\
0.016 \\
0.000 \\
0.814 \\
0.241 \\
0.004 \\
5.028\end{array}$ & $\begin{array}{l}2.751 \\
1.216 \\
0.012 \\
0.000 \\
0.242 \\
0.823 \\
0.007 \\
5.050\end{array}$ \\
\hline $\begin{array}{l}\text { An } \\
\mathrm{Ab} \\
\mathrm{Or}\end{array}$ & $\begin{array}{r}64.3 \\
35.4 \\
0.3\end{array}$ & $\begin{array}{r}71.4 \\
28.4 \\
0.2\end{array}$ & $\begin{array}{r}72.6 \\
27.4 \\
0.0\end{array}$ & $\begin{array}{r}54.3 \\
44.9 \\
0.8\end{array}$ & $\begin{array}{r}77.9 \\
21.9 \\
0.1\end{array}$ & $\begin{array}{r}26.5 \\
72.7 \\
0.8\end{array}$ & $\begin{array}{r}76.9 \\
22.8 \\
0.3\end{array}$ & $\begin{array}{r}22.6 \\
76.8 \\
0.7\end{array}$ \\
\hline
\end{tabular}

\begin{tabular}{|c|c|c|c|c|c|c|c|}
\hline \multirow[t]{2}{*}{$\begin{array}{l}\text { Hole: } \\
\text { Core, section: } \\
\text { Interval }(\mathrm{cm}) \text { : } \\
\text { Rock type: } \\
\text { * }\end{array}$} & \multicolumn{2}{|c|}{$\begin{array}{c}829 \mathrm{~A} \\
44 \mathrm{R}-1 \\
1-2 \\
\text { Serpentinite }\end{array}$} & \multicolumn{2}{|c|}{$\begin{array}{c}829 \mathrm{~A} \\
59 \mathrm{R}-1 \\
81-86 \\
\text { Dolerite } \\
\end{array}$} & \multicolumn{2}{|c|}{$\begin{array}{c}829 A \\
61 R-1 \\
1-3 \\
\text { Gabbro } \\
\end{array}$} & \multirow{2}{*}{$\begin{array}{c}829 \mathrm{~A} \\
61 \mathrm{R}-1 \\
36-38 \\
\text { Microgabbro } \\
\mathrm{mt}\end{array}$} \\
\hline & A sp & B sp & $\mathrm{Amt}$ & $\mathrm{B} \mathrm{mt}$ & A mt & $\mathrm{B} \mathrm{mt}$ & \\
\hline $\begin{array}{l}\mathrm{SiO}_{2} \\
\mathrm{TiO}_{2} \\
\mathrm{Al}_{2} \mathrm{O}_{3} \\
\mathrm{Fe}_{2} \mathrm{O}_{3} \\
\mathrm{FeO} \\
\mathrm{MnO} \\
\mathrm{MgO} \\
\mathrm{CaO} \\
\mathrm{Cr}_{2} \mathrm{O}_{3} \\
\mathrm{NiO} \\
\mathrm{Tot}\end{array}$ & $\begin{array}{r}0.00 \\
0.31 \\
28.55 \\
3.02 \\
15.76 \\
0.27 \\
13.39 \\
0.00 \\
39.20 \\
0.26 \\
100.75\end{array}$ & $\begin{array}{r}0.00 \\
0.23 \\
28.99 \\
2.39 \\
16.99 \\
0.18 \\
12.18 \\
0.00 \\
36.70 \\
0.08 \\
97.73\end{array}$ & $\begin{array}{r}0.74 \\
3.09 \\
0.25 \\
63.16 \\
33.53 \\
0.26 \\
0.21 \\
0.11 \\
0.05 \\
0.07 \\
101.47\end{array}$ & $\begin{array}{r}1.36 \\
13.53 \\
0.79 \\
41.81 \\
43.37 \\
0.28 \\
0.03 \\
0.27 \\
0.02 \\
0.08 \\
101.53\end{array}$ & $\begin{array}{r}0.08 \\
21.28 \\
1.74 \\
26.52 \\
49.34 \\
0.80 \\
0.72 \\
0.00 \\
0.03 \\
0.01 \\
100.53\end{array}$ & $\begin{array}{r}0.17 \\
14.58 \\
2.06 \\
37.62 \\
43.26 \\
0.80 \\
0.30 \\
0.07 \\
0.00 \\
0.03 \\
98.89\end{array}$ & $\begin{array}{r}0.09 \\
19.41 \\
2.11 \\
29.90 \\
47.02 \\
0.64 \\
1.22 \\
0.00 \\
0.00 \\
0.10 \\
100.48\end{array}$ \\
\hline $\begin{array}{l}\mathrm{Si} \\
\mathrm{Ti} \\
\mathrm{Al} \\
\mathrm{Fe}^{3+} \\
\mathrm{Fe}^{2+} \\
\mathrm{Mn} \\
\mathrm{Mg} \\
\mathrm{Ca} \\
\mathrm{Cr} \\
\mathrm{Ni} \\
\mathrm{Tot}\end{array}$ & $\begin{array}{r}0.000 \\
0.041 \\
6.009 \\
0.406 \\
2.354 \\
0.041 \\
3.563 \\
0.000 \\
5.534 \\
0.037 \\
17.985\end{array}$ & $\begin{array}{r}0.000 \\
0.031 \\
6.289 \\
0.331 \\
2.615 \\
0.028 \\
3.341 \\
0.000 \\
5.341 \\
0.012 \\
17.989\end{array}$ & $\begin{array}{l}0.028 \\
0.087 \\
0.011 \\
1.782 \\
1.051 \\
0.008 \\
0.012 \\
0.004 \\
0.002 \\
0.002 \\
2.988\end{array}$ & $\begin{array}{l}0.050 \\
0.375 \\
0.035 \\
1.159 \\
1.336 \\
0.009 \\
0.002 \\
0.011 \\
0.001 \\
0.002 \\
2.978\end{array}$ & $\begin{array}{l}0.003 \\
0.591 \\
0.076 \\
0.738 \\
1.525 \\
0.025 \\
0.040 \\
0.000 \\
0.001 \\
0.000 \\
2.999\end{array}$ & $\begin{array}{l}0.006 \\
0.415 \\
0.092 \\
1.071 \\
1.368 \\
0.026 \\
0.017 \\
0.003 \\
0.000 \\
0.001 \\
2.998\end{array}$ & $\begin{array}{l}0.003 \\
0.538 \\
0.092 \\
0.829 \\
1.448 \\
0.020 \\
0.067 \\
0.000 \\
0.000 \\
0.003 \\
2.999\end{array}$ \\
\hline $\begin{array}{l}\text { mgv } \\
\text { crv } \\
\text { Mag } \\
\text { Usp } \\
\text { Jcb }\end{array}$ & $\begin{array}{l}0.60 \\
47.9\end{array}$ & $\begin{array}{c}0.56 \\
45.9\end{array}$ & $\begin{array}{r}88.4 \\
8.7 \\
0.8\end{array}$ & $\begin{array}{r}58.0 \\
37.8 \\
0.9\end{array}$ & $\begin{array}{r}34.3 \\
59.2 \\
2.5\end{array}$ & $\begin{array}{r}52.0 \\
41.5 \\
2.6\end{array}$ & $\begin{array}{r}38.7 \\
53.8 \\
2.0\end{array}$ \\
\hline
\end{tabular}

Notes: $\mathrm{An}, \mathrm{Ab}$, and $\mathrm{Or}$ indicate anorthite, albite, and orthoclase percentages. Mag, Usp, and Jcb indicate magnetite, ulvospinel, and jacobsite molecule percentages, respectively; $\mathrm{crv}=\mathrm{Cr} / \mathrm{Cr}+\mathrm{Al}$ (at\%). *Abbreviations: $\mathrm{pl}=$ plagioclase; $\mathrm{sp}=$ spinel; $\mathrm{mt}=$ magnetite. Other abbreviations as in Table 2.

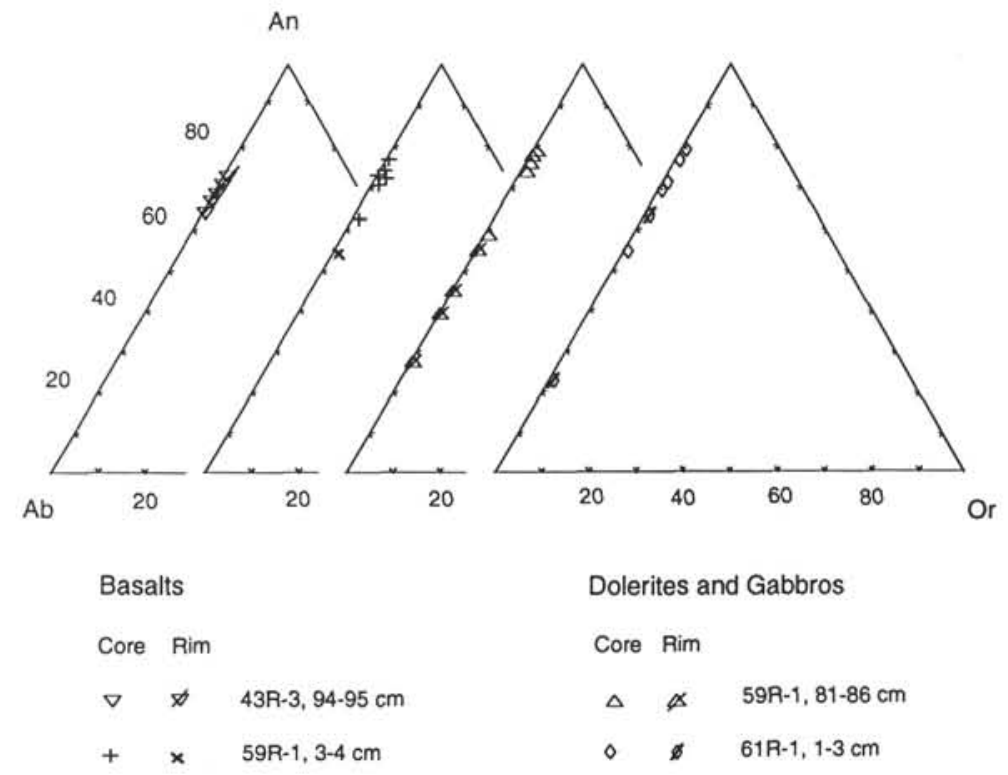

Figure 6. Plagioclase compositions of basalts, dolerites, and gabbros recovered from Hole $829 \mathrm{~A}$. Compositions of microphenocrysts and groundmass crystals are indicated as core and rim, respectively.

Sun et al., 1979; Sun, 1980; Sun and McDonough, 1989). Their MORB-normalized incompatible element patterns show, in some cases, a weak HFSE depletion (Figs. 9B and C). REE patterns for basalts and gabbros display moderate to marked LREE depletion (Fig. 10), (basalts and dolerites, $[\mathrm{La} / \mathrm{Yb}]_{\mathrm{n}}=0.46-0.48$; gabbros and microgabbros $\left.[\mathrm{La} / \mathrm{Yb}]_{\mathrm{n}}=0.13-0.27\right)$, even more pronounced than for normal MORB.

The few $\mathrm{Pb}$ isotopic data available for Site 829 (Table 5) are reported in Figure 11, together with the field of MORB from the Indian, Pacific, and Atlantic oceans and lavas from several island arcs (data from Sun, 1980; White and Dupré, 1986; Wilson, 1989). Site 829 gabbros and basalts plot outside the MORB fields, toward more radiogenic $\mathrm{Pb}$ values. They have higher ${ }^{207} \mathrm{~Pb} /{ }^{204} \mathrm{~Pb}$ values than the few data available for volcanics of the Central Chain of the New Hebrides Island Arc (Briqueu et al., this volume). Basaltic rocks from Site 828 (Coltorti et al., this volume) also show a tendency toward more radiogenic $\mathrm{Pb}$ values, comparable with those of Site 829 volcanics.

The serpentinite clast (Sample 134-829A-44R-1,3-4 cm) has high $\mathrm{MgO}, \mathrm{Ni}$, and $\mathrm{Cr}$ contents, and $\mathrm{mgv}=0.92$ (Table 1). Although a variation in $\mathrm{CaO}$ content due to alteration cannot be ruled out, the 
Table 5. Sr, Nd, and $\mathrm{Pb}$ isotopic compositions of some igneous rocks from Sites 829 and 830 .

\begin{tabular}{lccccccc}
\hline Hole: & $829 \mathrm{~A}$ & $829 \mathrm{~A}$ & $829 \mathrm{~A}$ & $829 \mathrm{~A}$ & $829 \mathrm{~A}$ & $830 \mathrm{~B}$ & $830 \mathrm{~B}$ \\
Core, section: & $43 \mathrm{R}-3$ & $59 \mathrm{R}-1$ & $59 \mathrm{R}-1$ & $61 \mathrm{R}-1$ & $64 \mathrm{R}-1$ & $14 \mathrm{R}-1$ & $14 \mathrm{R}-1$ \\
Interval $(\mathrm{cm}):$ & $130-133$ & $1-3$ & $122-124$ & $41-43$ & $13-16$ & $13-17$ & $47-51$ \\
\hline${ }^{87} \mathrm{Sr} /{ }^{86} \mathrm{Sr}$ & $0.70450(2)$ & $0.70580(1)$ & $0.70320(7)$ & $0.70325(4)$ & & $0.70379(4)$ & $0.70331(13)$ \\
${ }^{143} \mathrm{Nd} /{ }^{144} \mathrm{Nd}$ & & & & $0.51307(1)$ & $0.51317(2)$ & & \\
${ }^{206} \mathrm{~Pb} / 04 \mathrm{~Pb}$ & $18.727(4)$ & $18.865(4)$ & $18.602(4)$ & $18.569(5)$ & $18.616(8)$ & $18.696(3)$ & $18.754(3)$ \\
${ }^{207} \mathrm{~Pb} / 204 \mathrm{~Pb}$ & $15.550(4)$ & $15.659(4)$ & $15.609(4)$ & $15.601(5)$ & $15.619(8)$ & $15.556(4)$ & $15.575(4)$ \\
${ }^{208} \mathrm{~Pb} /{ }^{204} \mathrm{~Pb}$ & $38.248(12)$ & $38.862(12)$ & $38.500(13)$ & $38.474(14)$ & $38.547(22)$ & $38.388(12)$ & $38.570(11)$ \\
\end{tabular}

Note: Precision for isotopic data, expressed as 2 sigma, is reported in brackets.

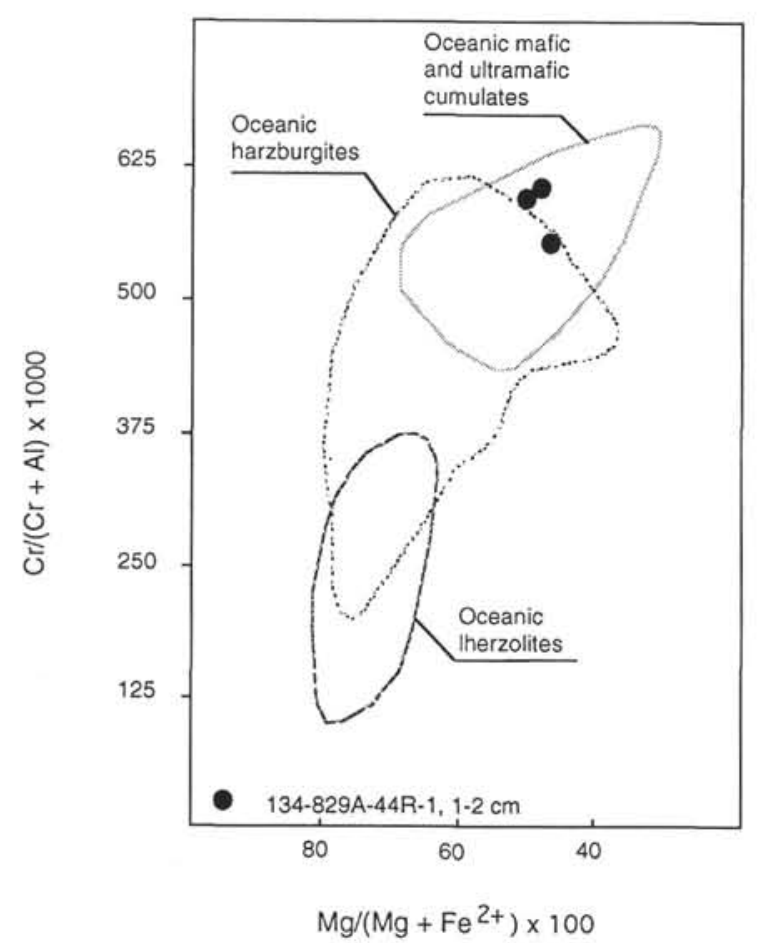

Figure 7. $\mathrm{Cr} /(\mathrm{Cr}+\mathrm{Al})($ at $\% \times 1000)$ vs. $\mathrm{Mg} / \mathrm{Mg}+\mathrm{Fe}^{2+}(\times 100)$ of spinels from serpentinite from Site 829. Fields of oceanic lherzolites, harzburgites, and mafic and ultramafic cumulates are from Hebert et al. (1989).

$\mathrm{CaO} / \mathrm{Al}_{2} \mathrm{O}_{3}$ value $(0.81)$ approaches estimates for primordial mantle composition (0.88, Jagoutz et al., 1979; 0.79, Hofmann, 1988). Normative minerals calculated for this sample indicate a harzburgitic composition, with clinopyroxene less than $5 \mathrm{wt} \%$; a simple mass balance calculation using rock and mineral chemical analyses, produces a similar result, giving a clinopyroxene content of about $4 \mathrm{wt} \%$. A serpentinized ultramafic clast was also recently found at the toe of the accretionary wedge in front of the Bougainville Guyot during a submersible dive (Collot et al., 1992). It has an overall bulk composition very similar to that recovered at Site 829 .

\section{Site 830}

Volcanic rocks from Site 830 are moderately to highly hypersthene-normative (5.34-22.05 wt\%), and their LOI values range from 3.51 to $7.07 \mathrm{wt} \%$. All samples are basalts, with less than $52 \mathrm{wt} \%$ $\mathrm{SiO}_{2}$ and show a variable degree of fractionation, as reflected in mgv values from 0.68 to 0.49 . Samples $134-830 \mathrm{~B}-14 \mathrm{R}-1,13-17 \mathrm{~cm}$, and $-14 \mathrm{R}-1,59-64 \mathrm{~cm}$ (the highest in the stratigraphic sequences), are the least fractionated, having the highest mgv values and $\mathrm{Ni}, \mathrm{Co}$, and $\mathrm{Cr}$ contents. The lack of a Fe-enrichment trend is consistent with early magnetite fractionation.

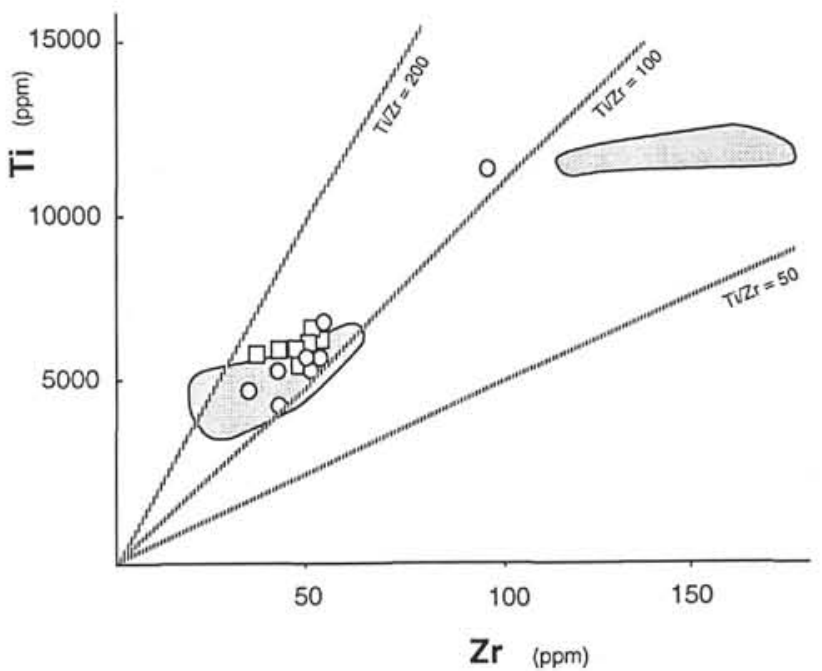

Figure 8. Ti vs. $\mathrm{Zr}$ contents in basalts and dolerites (open squares) and gabbros (open circles) from Site 829 . Fields of Site 828 basaltic rocks (hatched areas) are reported for comparison (Coltorti et al., this volume). Fields of island arc tholeiites, ocean ridge basalts, and calc-alkaline basalts are from Pearce and Cann (1973).

Chondrite-normalized REE patterns (Fig. 12A) and MORB-normalized incompatible element diagram (Fig. 12B) consistently show that the more fractionated rocks parallel, at higher values, the trend of the less differentiated samples, suggesting a genetic link among these lavas. All samples show distinct negative $\mathrm{Nb}$ anomalies, together with pronounced HFSE depletion (for the least fractionated samples), coupled with LFSE and LREE enrichments.

Lead isotopic ratios for two Site 830 samples (Table 5) plot in the fields for island arc lavas (Fig. 11), and are also very similar to the limited available data for basalts from islands of the New Hebrides Central Chain (Briqueu et al., this volume), although the provenance of Site 830 basaltic rocks is to be sought in the Western Belt islands.

\section{DISCUSSION}

\section{Site 827}

Rocks recovered at this site are mainly volcaniclastic/epiclastic material occurring below an upper Pliocene to middle Pleistocene volcanic siltstone. Clasts of highly plagioclase-phyric basalts, rare dacite, and volcanic breccias are present in a matrix made up of plagioclase, clinopyroxene, amphibole, opaques, and altered glass.

A thick sequence of late Oligocene to middle Miocene volcanoclastics occurs on Espiritu Santo Island (Santo Volcanic Group; Mallick and Greenbaum, 1977). Its sedimentological and petrographic characteristics are very similar to the deposits found at Site 827 . Hornblendebearing andesite is a widely distributed lithotype in the Santo Volcanic Group, followed by highly plagioclase-phyric andesite (up to $30 \mathrm{vol} \%$ 

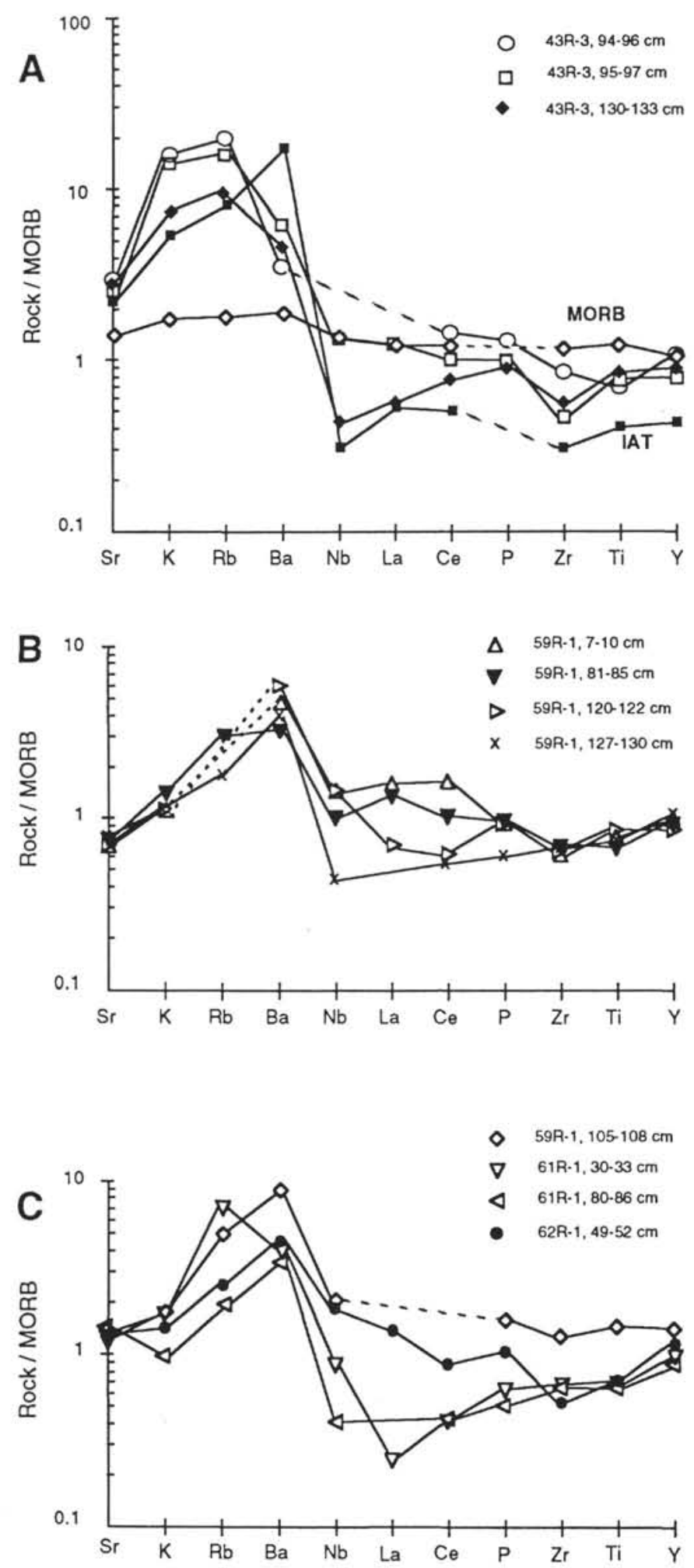

Figure 9. MORB-normalized incompatible element patterns for (A) basalts, (B) dolerites, and (C) gabbros and microgabbros from Hole 829A. Normalizing values from Sun and McDonough (1989). MORB and IAT are representative analyses from Sun (1980).

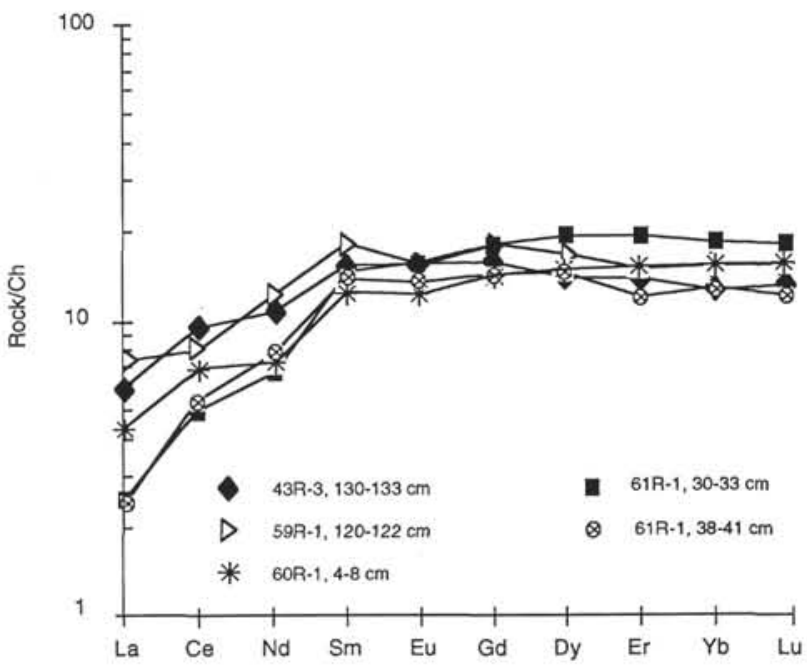

Figure 10. Chondrite-normalized REE distributions for basalts, dolerites, and gabbros from Hole 829A. Normalizing values from Sun and McDonough (1989).

of phenocrysts), basaltic andesite, basalts, and subordinate dacites. The petrography of these rocks matches very closely the igneous components of the volcanic breccia recovered at Site 827 , both as clasts and as crystals dispersed in the matrix. Clinopyroxene compositions and, particularly, the presence of amphibole phenocrysts in one andesitic clast suggest a calc-alkaline magmatic affinity.

These data, together with the proximal nature of the Site 827 deposit (very poorly sorted, with angular to subrounded clasts), suggest that this material most probably originated from the Western Belt of the New Hebrides Island Arc. At this stage, it is not possible to distinguish the relative contribution of primary pyroclastic and autoclastic materials on the one hand, from epiclastic materials on the other. The first generation breccia may have been produced by magmaseawater interaction-induced fragmentation of a single lava flow, because both clasts and matrix show similar parageneses and modal abundances (Jones, 1967). This volcaniclastic material was, in turn, emplaced as a rubble avalanche (Jones, 1967) or as submarine lahars (Mitchell, 1970) along the forearc slope of Espiritu Santo Island.

\section{Site 829}

Basalts, dolerites, gabbros, and ultramafic rocks occur at various levels in Site 829. Effusive rocks vary from sparsely clinopyroxene + plagioclase-phyric to moderately-plagioclase-phyric basalts. Clinopyroxene phenocrysts in these basalts have lower Ti contents relative to $\mathrm{Al}^{\mathrm{IV}}$ than those in MORB and are compositionally similar to clinopyroxenes from Site 828 (Coltorti et al., this volume). Basalts are quite primitive and have Ti contents intermediate between those in N-MORB and island arc basalts. Incompatible element distributions further support this transitional character; and HFSE levels are lower than MORB and higher than the average IAT values (Sun, 1980), whereas the reverse is true for LFSE. The latter elements, however, are unreliable, since they are easily mobilized during alteration (Alt et al., 1986; Bienvenu et al., 1990). The higher Ti/Zr values, generally lower HFSE levels, and steeper LREE-depletion compared with normal MORB all argue for a source peridotite that was rather more refractory than for normal MORB (Woodhead et al., 1993), or alternatively, that they were derived by higher extents of partial melting of MORB-source peridotite than that which yields typical MORB.

Despite a strong leaching procedure, ${ }^{87} \mathrm{Sr} /{ }^{86} \mathrm{Sr}$ isotopic ratios vary from 0.7045 to 0.7058 (Table 5), values significantly higher than for N-MORB (DePaolo, 1988). Furthermore, on a ${ }^{207} \mathrm{~Pb} /{ }^{204} \mathrm{~Pb}$ vs. ${ }^{206} \mathrm{~Pb} /{ }^{204} \mathrm{~Pb}$ diagram, Site 829 basalts plot at the limit or well outside 


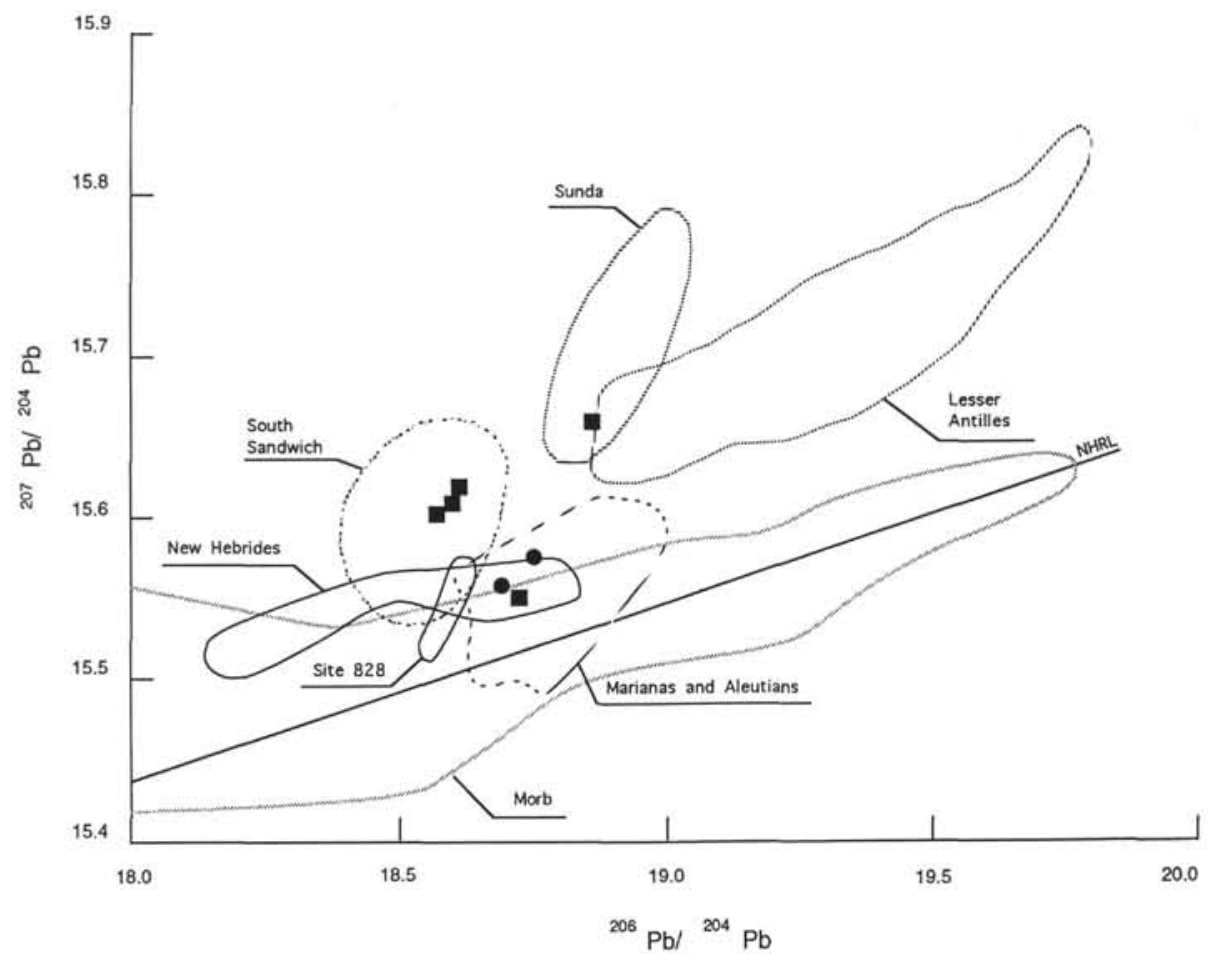

Figure 11. Lead isotopic ratios of basaltic and gabbroic rocks from Sites 829 (filled squares) and 830 (filled circles). Field of basaltic rocks from Site 828 (NDR) (Coltorti et al., this volume) and Central Chain of New Hebrides islands (Briqueu et al., this volume) are reported for comparison. Fields of basaltic rocks from island arc and mid-ocean ridge settings are from Sun (1980), White and Dupré (1986), and Wilson (1989). NHRL $=$ North Hemisphere Reference Line.

the MORB fields toward more radiogenic values, characteristic of arc basalts.

Dolerites, microgabbros, and gabbros show petrographic and geochemical features similar to those of basalts. The crystallization sequence in these rocks (i.e., plagioclase crystallizing before clinopyroxene) is typical of MORB-type basalts, although some small crystals of biotite found in gabbros suggest a $\mathrm{K}_{2} \mathrm{O}$ content higher than in normal-MORB (Prichard and Cann, 1982; Spadea et al., 1991). Tholeiitic differentiation trends with strong $\mathrm{FeO}$-enrichment are clearly recorded in the olivine and clinopyroxene geochemistry. Ti vs. $\mathrm{Al}^{\mathrm{IV}}$ contents in clinopyroxenes mostly plot in the IAT field. Chondritenormalized minor and trace element distribution vary from nearly flat (except for $\mathrm{Rb}$ and $\mathrm{Ba}$ ) to slightly depleted in HFSE and enriched in LFSE (Fig. 9C). ${ }^{87} \mathrm{Sr} /{ }^{86} \mathrm{Sr}$ isotopic ratios are in the MORB range and the single Nd determination plots inside the MORB field. In contrast ${ }^{207} \mathrm{~Pb} /{ }^{04} \mathrm{~Pb}$ vs. ${ }^{206} \mathrm{~Pb} /{ }^{204} \mathrm{~Pb}$ isotopic ratios fall within the field of South Sandwich arc volcanics, and are more radiogenic than normal-MORB. These compositional features taken together suggest that the Site 829 basalts and comagmatic dolerites and gabbros probably represent backarc basin basalt magmatic affinities. Effusive rocks with similar intermediate characteristics between MORB and IAT were drilled in the Sulu Sea, a small intra-oceanic basin behind the Sulu Arc (ODP Leg 124; Spadea et al., 1991) and classified as back-arc basin basalts (BABB), by analogy with basaltic rocks found in the North Fiji Basin (Price et al., 1990) and Mariana Trough (Sinton and Fryer, 1987).

The origin of the serpentinite clast cored at around $407 \mathrm{mbsf}$ at Site 829 is difficult to determine. It was originally harzburgitic. However available mineral chemical data cannot determine whether it formed as a residual tectonite peridotite during extraction of tholeiitic magma from sub-oceanic upper mantle, or whether it was a cumulate from a primitive magma. The latter hypothesis seems unlikely, since the only magmas that crystallize significant amounts of orthopyroxene before clinopyroxene are those with boninitic affini- ties (Crawford et al., 1989), and boninitic magmatism is unknown and unexpected in the region.

On Espiritu Santo and Malakula islands basalts, basaltic andesites, and andesites occur, either as isolated flows or as clasts in volcaniclastic breccias, together with minor intrusion of quartz diorite, diorites, and gabbro. Hornblende is always a common phase in both the effusive and intrusive rocks (Mallick and Greenbaum, 1977; Macfarlane and Carney, 1987). The few available data from Espiritu Santo (Mallick and Greenbaum, 1977), Malakula (Macfarlane and Carney, 1987), and Torres islands indicate a transitional calc-alkaline/tholeiitic character (Macfarlane et al., 1988), partly resembling the lower- $\mathrm{K}_{2} \mathrm{O}$ suite of the Central Chain lavas (Crawford et al., 1988). The only two trace element analyses of gabbro and diorite from Malakula (Gorton, 1974) show higher $\mathrm{Sr}, \mathrm{Rb}, \mathrm{K}, \mathrm{Ba}, \mathrm{La}$, and $\mathrm{Ce}$ and lower $\mathrm{Zr}$, Ti, and $\mathrm{Y}$ contents with respect to Site 829 igneous rocks. These two analyses, reported on a MORB-normalized diagram, display a clear $\mathrm{Nb}$ negative anomaly (Fig. 12). Clearly petrography and geochemistry of igneous rocks from the Western Belt islands are quite unlike from Site 829. On the other hand, basaltic rocks whose petrological features compare favorably with those drilled on the forearc are found on the NDR (Coltorti et al., this volume; Fig. 13). This strongly suggests that the forearc rocks drilled at Site 829 have been accreted to the over-riding plate during collision of the NDR with the forearc of this central section of the New Hebrides volcanic arc.

Concerning the provenance of the peridotitic clast, the only known outcrop of ultramafic rocks in the Vanuatu Archipelago is on the eastern side of Pentecost Island (Mallick and Neef, 1974). However, if the clast really came from this island, erosion and transport before the uplift of Espiritu Santo and Malakula islands should be considered (Collot et al., 1992). In our opinion, two alternative hypotheses can be put forward: (1) exhumation of mantle material by deep thrust plane, affecting the over-riding Pacific Plate at the convergent margin (Collot et al., 1992); or (2) accretion/obduction of mantle material onto the 

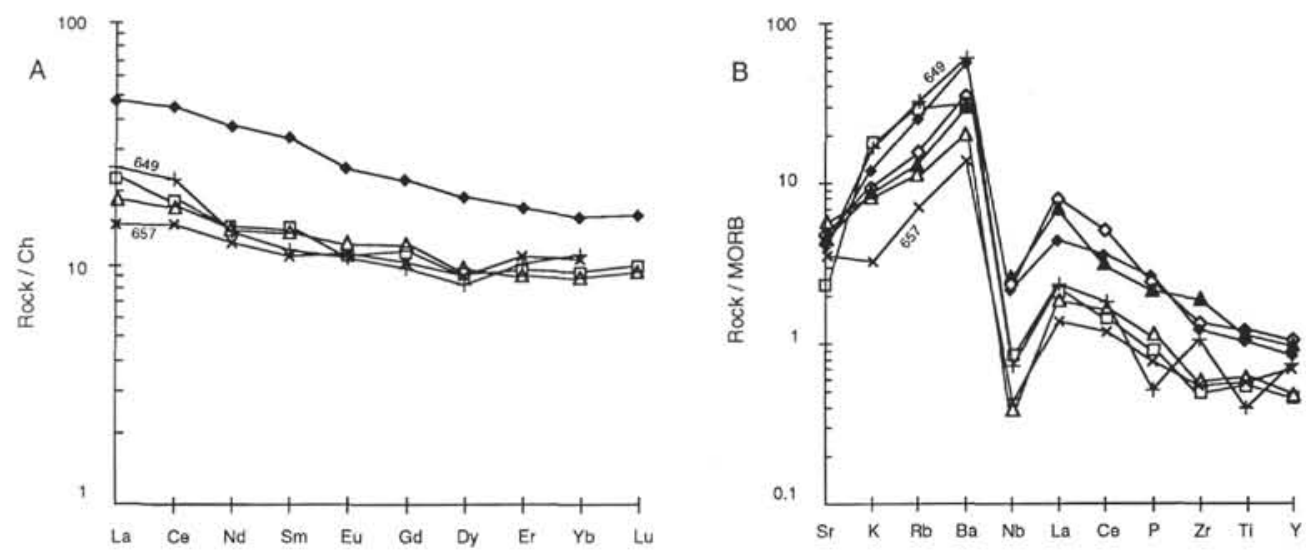

$\triangle 14 \mathrm{R}-1,13-17 \mathrm{~cm}$

ㅁ $14 \mathrm{R}-1,59-64 \mathrm{~cm}$

A $22 \mathrm{R}-1,32-33 \mathrm{~cm}$

- $22 \mathrm{~A} \cdot 1,34-37 \mathrm{~cm}$

- $22 \mathrm{R}-1,40-44 \mathrm{~cm}$

Figure 12. Basalts from Hole 830B. A. Chondrite-normalized REE patterns. B. MORB-normalized incompatible element distribution. 657 and 649 are two samples (gabbro and diorite, respectively) from Malakula Island (Gorton, 1974). Normalizing values from Sun and McDonough (1989).

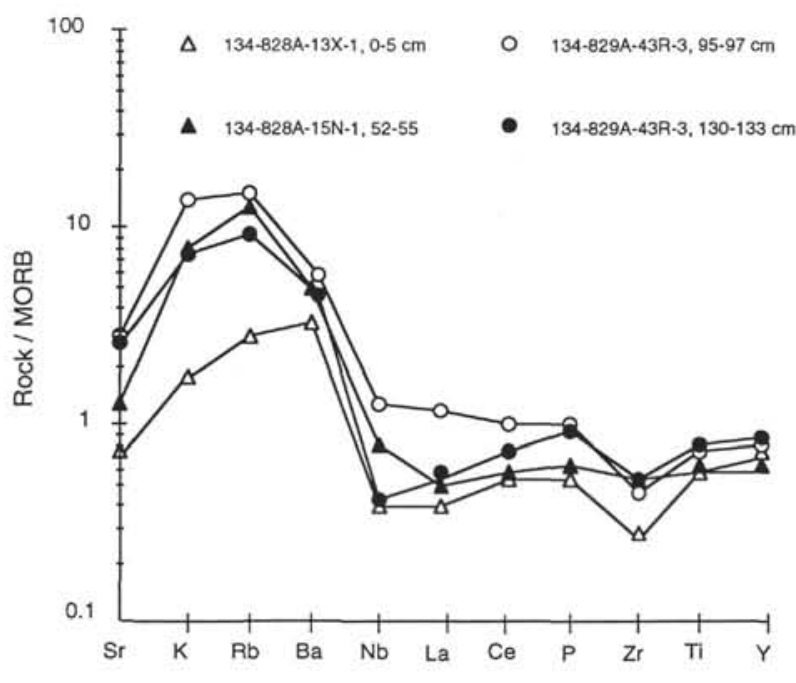

Figure 13. MORB-normalized incompatible element patterns of basaltic rocks from Site 829 compared with basaltic lavas from Site 828 (NDR; Coltorti et al., this volume).

DEZ after the Eocene subduction event (Maillet et al., 1983; Kroenke, 1984), accompanied by subsequent accretion on the forearc region of the New Hebrides Arc.

\section{Site 830}

Basalts recovered as clasts at Site 830 are strongly hypersthenenormative, and vary from quite primitive to fairly differentiated magmas $(\mathrm{mgv}=0.68-0.49)$. They are found below Pleistocene volcanic silt/siltstone. Petrography of these clasts (both lavas and breccias), together with type and modal proportion of crystal fragments in the matrix, resemble those found in the volcaniclastic/epiclastic deposit of Site 827 . Sedimentary and volcanic structures also suggest similar depositional processes.

Trace element distributions show the following: (1) a remarkable HFSE depletion coupled with evident LFSE enrichment; (2) a pronounced Nb-negative anomaly; and (3) an LREE enrichment coupled with flat unfractionated HREE. In the MORB-normalized diagram of Figure 12, the parallelism with the two samples from Malakula Island (Gorton, 1974) is evident. Lead isotopic data for these samples are similar to those of volcanics from the Central Chain of the New Hebrides Island Arc, indicating a clear island arc magmatic affinity.

The age and nature of the deposit suggest that these lavas are derived from the nearby Western Belt volcanic islands of Vanuatu. As for Site 827, these rocks probably represent reworked material from a volcaniclastic formation. From their locations Site 830 sediments are probably derived from the southern part of Espiritu Santo Island and/or northern Malakula Island. However, it should be noted that, in addition to the close similarity in trace element patterns of the basaltic rocks, hornblende (particularly as crystal fragments in Site 830 igneous rocks), is less abundant than in rocks from Site 827 . These findings suggest a greater contribution of material from Malakula Island in the Site 830 deposits with respect to those of Site 827.

On Malakula Island, abundant volcanic breccias petrographically similar to the rocks drilled at Site 830 occur in the Matanui Formation, of lower Miocene age (Mitchell, 1966). The similarity of basalt compositions to those on nearby Malakula, plus the paucity of detrital hornblende relative to those in the sequence at Site 827 , suggest a greater contribution of detritus from Malakula Island in the Site 830 deposits with respect to those of Site 827 .

\section{CONCLUSIONS}

1. Site 827 recovered coarse volcaniclastic and epiclastic reworked lava breccias beneath upper Pliocene volcaniclastic siltstone. Clasts of basalt and subordinate dacite occur in a matrix that contains abundant detrital plagioclase, augite, amphibole, and altered vitric ash. Based on compositions of phenocryst minerals, and particularly the presence of amphibole, these rocks are considered to be derived from calc-alkaline lavas. Petrographically identical lavas occur in the late Oligocene to middle Miocene Santo Volcanic Group on Espiritu Santo Island, some $35 \mathrm{~km}$ further east, and the Site 827 rocks are inferred to have derived from this source area and may have emplaced as submarine lahars and rubble avalanches.

2. Site 829 recovered basalts and comagmatic dolerites and gabbroic rocks, plus a single clast of serpentinized harzburgite in a faultdisrupted sequence of rocks ranging from pre-middle Oligocene to Pleistocene age. Major and trace element characteristics of the mafic igneous rocks are transitional between typical normal MORB and island arc tholeiites, and $\mathrm{Pb}$ and $\mathrm{Sr}$ isotopic ratios are more radiogenic than typical MORB. Petrographically and compositionally very similar rocks occur at Site 828 on the North d'Entrecasteaux Ridge (Fig. 13). We conclude that the rocks drilled at Site 829 were accreted into the forearc of the New Hebrides Island Arc during collision of the DEZ with the arc. 
3. Site 830 , located in the forearc between the colliding Bougainville Guyot and the central New Hebrides Island Arc, yielded coarse volcaniclastic and epiclastic rocks below Pleistocene volcaniclastic silts. Clasts in these rocks have major element, trace element, and $\mathrm{Pb}$ isotopic compositions similar to New Hebrides Island Arc lavas that pre-date the collision of the d'Entrecasteaux Ridge. Volcanic breccias of the lower Miocene Matanui Formation of Malakula Island may be the source of the Site 830 volcaniclastics.

\section{ACKNOWLEDGMENTS}

M. Coltorti's participation on Leg 134 was supported by C.N.R. (Italy). The authors thank E. Condliffe, A. Fujinawa, and C. LaPorte for support in the analytical work. L. Beccaluva and F. Siena provided valuable suggestions. Constructive reviews by Steve Eggins, Julian Pearce, and Tony Crawford greatly improved the early version of the manuscript. We thank Tony Crawford for the invaluable overviews of the complex geological evolution of our studied area.

\section{REFERENCES ${ }^{*}$}

Alt, J.C., Honnorez, J., Laverne, C., and Emmermann, R., 1986. Hydrothermal alteration of a $1 \mathrm{~km}$ section through the upper oceanic crust, Deep Sea Drilling Project Hole 504B: mineralogy, chemistry, and evolution of seawater-basalt interactions. J. Geophys. Res., 91:10309-10335.

Aubouin, J., 1989. Some aspects of the tectonics of subduction zones. Tectonophysics, 160:1-21.

Ballance, P.F., Scholl, D.W., Vallier, T.L., Stevenson, A.J., Ryan, H., and Herzer, R.H., 1989. Subduction of a Late Cretaceous seamount of the Louisville ridge at the Tonga trench: a model of normal and accelerated tectonic erosion. Tectonics, 8:953-962.

Beccaluva, L., Macciotta, G., Piccardo, G.B., and Zeda, O., 1989. Clinopyroxene composition of ophiolite basalts as petrogenetic indicator. Chem. Geol., 77:165-182.

Bienvenu, P., Bougault, H., Joron, J.L., Treuil, M., and Dimitriev, L., 1990. MORB alteration: rare-earth element/non-rare-earth hygromagmaphile element fractionation. Chem. Geol., 82:1-14.

Collot, J.-Y., and Fisher, M.A., 1988. Crustal structure, from gravity data, of a collision zone in the central New Hebrides Island Arc. In Greene, H.G., and Wong, F.L. (Eds.), Geology and Offshore Resources of Pacific Island Arcs-Vanuatu Region. Circum-Pac. Counc. Energy Miner. Resour., Earth Sci. Ser., 8:125-140.

Collot, J.-Y., Greene, H.G., Stokking, L.B., et al., 1992. Proc. ODP, Init. Repts., 134: College Station, TX (Ocean Drilling Program).

Collot, J.-Y, Lallemand, S., Pelletier, B., Eissen, J.-P., Glaçon, G., Fisher, M.A., Greene, H.G., Boulin, J., Daniel, J., and Monzier, M., 1992. Geology of the d'Entrecasteaux-New Hebrides island arc collision: results from a deep-sea submersible survey. Tectonophysics, 212:213-241.

Crawford, A.J., Falloon, T.J., and Green, D.H., 1989. Classification, petrogenesis and tectonic setting of boninites. In Crawford, A.J. (Ed.), Boninites and Related Rocks: London (Unwin Hyman), 1-49.

Crawford, A.J., Greene, H.G., and Exon, N.J., 1988. Geology, petrology and geochemistry of submarine volcanoes around Epi Island, New Hebrides Island Arc. In Greene, H.G., and Wong, F.L. (Eds.), Geology and Offshore Resources of Pacific Island Arcs-Vanuatu Region. Circum-Pac. Counc. Energy Miner. Resour., 8:301-327.

DePaolo, D., 1988. Neodymium Isotope Geochemistry: An Introduction: Berlin (Springer-Verlag).

Fisher, M.A., Collot, J.-Y., and Geist, E.L., 1991. The collision zone between the North d'Entrecasteaux Ridge and the New Hebrides Island Arc. Part 2: structure from multichannel seismic data. J. Geophys. Res., 96:4479-4495.

Franzini, M., Leoni, L., and Saitta, M., 1975. Revisione di una metodologia analitica per fluorescenza-X, basata sulla correzione completa degli effetti di matrice. Rend. Soc. Ital. Mineral. Petrol., 31:365-378.

Gorton, M.P., 1974. The geochemistry and geochronology of the New Hebrides [Ph.D. dissert.]. Australian National Univ., Canberra.

\footnotetext{
Abbreviations for names of organizations and publications in ODP reference lists follow the style given in Chemical Abstracts Service Source Index (published by American Chemical Society).
}

Hebert, R., Serri, G., and Hékinian, R., 1989. Mineral chemistry of ultramafic tectonites and ultramafic to gabbroic cumulates from the major oceanic basins and the Northern Apennines ophiolites (Italy): a comparison. Chem. Geol., 77:183-207.

Hofmann, A.W., 1988. Chemical differentiation of the Earth: the relationship between mantle, continental crust, and oceanic crust. Earth Planet. Sci. Lett., 90:297-314.

Jagoutz, E., Palme, H., Baddenhausen, H., Blum, K., Cendales, M., Dreibus, G., Spettel, B., Lorenz, V., and Wanke, H., 1979. The abundances of major, minor and trace elements in the Earth's mantle as derived from primitive ultramafic nodules. Proc. 10th Lunar Planet. Sci. Conf., 2031-2050.

Jones, J.G., 1967. Clastic rocks of Espiritu Santo Island, New Hebrides. Bull. Geol. Soc. Am., 78:1281-1288.

Karig, D.E., and Sharman, G.F., III, 1975. Subduction and accretion in trenches. Geol. Soc. Am. Bull., 86:377-389.

Kobayashi, K., Cadet, J.-P., Aubouin, J., Boulègue, J., Dubois, J., von Huene, R., Jolivet, L., Kanazawa, T., Kasahara, J., Koisumi, K., Lallemand, S., Nakamura, Y.P., Suyehiro, K., Tani, S., Tokuyama, H., and Yamazaki, T., 1987. Normal faulting of the Daiiche Kashima seamount in the Japan trench revealed by the Kaiko I cruise, Leg 3. Earth Planet. Sci. Lett., $83: 257-266$.

Kroenke, L., 1984. Cenozoic Development of the Southwest Pacific. U.N. Econ. Soc. Comm. Asia Pac., Suva, Fiji. CCOP/SOPAC, Tech. Bull., 6.

Lallemand, S., Culotta, R., and von Huene, R., 1989. Subduction of the Daiichi Kashima seamount in the Japan trench. Tectonophysics, 160:231-248.

Leoni, L., and Saitta, M., 1976. X-ray flourescence analyses of 29 trace elements in rock and mineral standards. Rend. Soc. Ital. Mineral. Petrol., 32:497-510.

Leterrier, J., Maury, R.C., Thonon, P., Girard, D.. and Marchal, M., 1982. Clinopyroxene composition as a method of identification of the magmatic affinities of paleo-volcanic series. Earth Planet. Sci. Lett., 59:139-154.

Macfarlane, A., and Carney, J.N., 1987. Geology and Mineralisation of North and Central Malakula. Vanuatu Dept. of Geol., Mines and Rural Water Supplies.

Macfarlane, A., Carney, J.N., Crawford, A.J., and Greene, H.G., 1988. Vanuatu-a review of the onshore geology. In Greene, H.G., and Wong, F.L. (Eds.), Geology and Offshore Resources of Pacific Island Arcs-Vanuatu Region. Circum-Pac. Counc. Energy Miner. Resour., Earth Sci. Ser., 8:45-91.

Maillet, P., Monzier, M., Selo, M., and Storzer, D., 1983. The d'Entrecasteaux zone (southwest Pacific). A petrological and geochronological reappraisal. Mar. Geol., 53:179-197.

Mallick, D.I.J., and Greenbaum, D., 1977. Geology of Southern Santo. Reg. Rep.- New Hebrides Condominium Geol. Surv.

Mallick, D.I.J., and Neef, G., 1974. Geology of Pentecost. Reg. Rep.-New Hebrides Geol. Surv.

Mitchell, A.H.G., 1970. Facies of an early Miocene volcanic arc, Malekula Island, New Hebrides. Sedimentology, 16:201-243.

- 1966. Geology of South Malakula. Rep. - New Hebrides Geol. Surv., 3.

Pearce, J.A., 1983. The role of sub-continental lithosphere in magma genesis at active continental margins. In Hawkesworth, C.J., and Norry, M.J. (Eds.), Continental Basalts and Mantle Xenoliths: Nantwich (Shiva Publ.), 230-249.

Pearce, J.A., and Cann, J.R., 1973. Tectonic setting of basic volcanic rocks determined using trace element analyses. Earth Planet. Sci. Lett., 19:290-300.

Price, R.C., Johnson, L.E., and Crawford, A.J., 1990. Basalts of the North Fiji Basin: the generation of back arc basin magmas by mixing of depleted and enriched mantle sources. Contrib. Mineral. Petrol., 105:106-121.

Prichard, H.M., and Cann, J.R., 1982. Petrology and mineralogy of dredged gabbro from Gettysburg Bank, Eastern Atlantic. Contrib. Mineral. Petrol., 79:46-55.

Reynolds, R.C., 1967. Estimations of mass absorption coefficients by Compton scattering: improvements and extensions of the method. Am. Mineral., 48:1133-1143.

Roelandts, I., and Michel, G., 1986. Sequential inductively coupled plasma determination of some rare-earth elements in five french geostandards. Geostand. Newsl., 10:135-154.

Scholl, D.W., von Huene, R., Vallier, T.L., and Howell, D.G., 1980. Sedimentary masses and concepts about tectonic processes at underthrust ocean margins. Geology, 8:564-568.

Serri, G., Hebert, R., and Hékinian, R., 1985. Chemistry of ultramafic tectonites and ultramafic to gabbroic cumulates from the major oceanic basins and the Northern Apennines ophiolites. Ofioliti, 10:63-76. 
Sinton, J.M., and Fryer, P., 1987. Mariana Trough lavas from $18^{\circ} \mathrm{N}$ : implications for the origin of back arc basin basalts. J. Geophys. Res., 92:12782-12802.

Spadea, P., Beccaluva, L., Civetta, L., Coltorti, M., Dostal, J., Sajona, F., Serri, G., Vaccaro, C., and Zeda, O., 1991. Petrology of basic igneous rocks from the floor of the Sulu Sea. In Silver, E.A., Rangin, C., von Breymann, M.T., et al., Proc. ODP, Sci. Results, 124: College Station, TX (Ocean Drilling Program), 251-270.

Sun, S.-S., 1980. Lead isotopic study of young volcanic rocks from mid-ocean ridges, ocean islands and island arcs. Philos. Trans. R. Soc. London A, 297:409-445.

Sun, S.-S., and McDonough, W.F., 1989. Chemical and isotopic systematics of oceanic basalts: implications for mantle composition and processes. In Saunders, A.D., and Norry, M.J. (Eds.), Magmatism in the Ocean Basins. Geol. Soc. Spec. Publ. London, 42:313-345.

Sun, S.-S., Nesbitt, R.W., and Sharaskin, A.Y., 1979. Geochemical characteristics of mid-ocean ridge basalts. Earth Planet. Sci. Lett., 44:119-138.
White, W.M., and Dupré, B., 1986. Sediment subduction and magma genesis in the Lesser Antilles: isotopic and trace element constraints. J. Geophys. Res., 91:5927-5941.

Wilson, M., 1989. Igneous Petrogenesis: A Global Tectonic Approach: London (Unwin Hyman).

Woodhead, J., Eggins, S., and Gamble, J., 1993. High field strength and transition element systematics in island arcs and back-arc basin basalts: evidence for multi-phase melt extraction and a depleted mantle wedge. Earth Planet. Sci. Lett., 114:491-504.

Yamazaki, T., and Okamura, Y., 1989. Subducting seamounts and deformation of overriding forearc wedges around Japan. Tectonophysics, 160:207-229.

Date of initial receipt: 24 April 1992

Date of acceptance: 17 June 1993

Ms 134SR-015 